\title{
Renormalon effects in quasiparton distributions
}

\author{
Wei-Yang Liu $\oplus^{1, *}$ and Jiunn-Wei Chen $\oplus^{1,2, \dagger}$ \\ ${ }^{1}$ Department of Physics, Center for Theoretical Physics, and Leung Center for Cosmology \\ and Particle Astrophysics, National Taiwan University, Taipei 106, Taiwan \\ ${ }^{2}$ Physics Division, National Center for Theoretical Sciences, National Taiwan University, \\ Taipei 10617, Taiwan
}

(Received 21 October 2020; accepted 13 September 2021; published 2 November 2021)

\begin{abstract}
We investigate the renormalon ambiguity from bubble-chain diagrams in the isovector unpolarized quasiparton distribution function (PDF) of a hadron. We confirm the assertion by Braun, Vladimirov, and Zhang [Phys. Rev. D 99, 014013 (2019)] that the leading IR renormalon ambiguity is an $\mathcal{O}\left(\Lambda_{\mathrm{QCD}}^{2} / x^{2} P_{z}^{2}\right)$ effect, with $x$ the parton momentum fraction and $P_{z}$ the hadron momentum, together with a new $\mathcal{O}\left(\delta(x) \Lambda_{\mathrm{QCD}}^{2} / P_{z}^{2}\right)$ contribution such that the quark number is conserved. This implies the convergence of the perturbative matching kernel between a quasi-PDF and a PDF would eventually fail for small $x$. However, in both the $R$-scheme designed to cancel the leading IR renormalon and the typically used regularization-independent momentum subtraction scheme in lattice QCD for the same quasi-PDF, we find good convergence in the kernel based on three-loop bubble-chain diagram analyses. These results are encouraging for the quasi-PDF program. However, firm conclusions can only be drawn after the complete higher loop QCD calculations are carried out.
\end{abstract}

DOI: 10.1103/PhysRevD.104.094501

\section{INTRODUCTION}

Large momentum effective theory (LaMET) enables computations of parton distributions of hadrons on a Euclidean lattice. LaMET relates equal-time spatial correlators (whose Fourier transforms are called quasidistributions) to light cone distributions in the infinite hadron momentum limit [1,2]. For large but finite momenta accessible on a realistic lattice, LaMET relates quasidistributions to physical ones through a factorization theorem, which involves a matching coefficient and power corrections that are suppressed by the hadron momentum. The proof of factorization was developed in Refs. [3-5].

Since LaMET was proposed, a lot of progress has been made in the theoretical understanding of the formalism [4,6-62]. The method has been applied in lattice calculations of parton distribution functions (PDFs) for the nucleon [20,26,27,29,63-76], $\pi$ [77-79], and $K$ [80] mesons. Despite limited volumes and relatively coarse lattice spacings, the state-of-the-art nucleon isovector quark PDFs, determined from lattice data at the physical point,

\footnotetext{
r07222052@g.ntu.edu.tw

jwc@phys.ntu.edu.tw
}

Published by the American Physical Society under the terms of the Creative Commons Attribution 4.0 International license. Further distribution of this work must maintain attribution to the author(s) and the published article's title, journal citation, and DOI. Funded by SCOAP . have shown reasonable agreement [66,67] with phenomenological results extracted from the experimental data. Encouraged by this success, LaMET has also been applied to $\Delta^{+}$[81] and twist-three PDFs [82-84], as well as gluon [85], strange, and charm distributions [86]. It was also applied to meson distribution amplitudes $[21,87,88]$ and generalized parton distributions (GPDs) [89-92]. More recently, attempts have been made to generalize LaMET to transverse momentum dependent (TMD) PDFs [93-100] to calculate the nonperturbative Collins-Soper evolution kernel $[95,101,102]$ and soft functions [103] on the lattice. LaMET also brought renewed interest in earlier approaches [104-110] and inspired new ones [111-126]. For recent reviews, see, e.g., Refs. [127-131].

The quark PDF in a proton is related to a light cone correlator

$$
\begin{aligned}
Q(x)= & \int_{-\infty}^{\infty} \frac{d \xi^{-}}{4 \pi} e^{-i x \xi^{-} P^{+}}\langle P| \bar{\psi}\left(\xi^{-}\right) \gamma^{+} \\
& \times \exp \left(-i g \int_{0}^{\xi^{-}} d \eta^{-} A^{+}\left(\eta^{-}\right)\right) \psi(0)|P\rangle,
\end{aligned}
$$

where the proton momentum $P^{\mu}$ is along the $z$ direction, $P^{\mu}=\left(P^{0}, 0,0, P^{z}\right)$, and $\xi^{ \pm}=(t \pm z) / \sqrt{2}$ is the light cone coordinate. $x$ is the parton momentum fraction relative to the proton. $A^{+}$is a gauge field and $\psi$ is a quark field. The expression in Eq. (1) is boost invariant along the $z$ direction. The corresponding quasi-PDF is related to an equal time correlator $[1,2]$ 


$$
\begin{aligned}
\tilde{Q}\left(x, P_{z}\right)= & \int_{-\infty}^{\infty} \frac{d z}{4 \pi} e^{i x z P z}\langle P| \bar{\psi}(z) \gamma^{z} \\
& \times \exp \left(-i g \int_{0}^{z} d z^{\prime} A^{z}\left(z^{\prime}\right)\right) \psi(0)|P\rangle .
\end{aligned}
$$

LaMET relates the flavor nonsinglet quark quasi-PDF and PDF of a hadron through the factorization theorem

$$
\tilde{Q}\left(x, P_{z}, \Lambda^{\prime}\right)=\int_{-1}^{1} \frac{d y}{|y|} Z\left(\frac{x}{y}, y P_{z}, \Lambda^{\prime}, \Lambda\right) Q(y, \Lambda)+\mathcal{O}\left(\frac{1}{P_{z}^{2}}\right),
$$

where $\Lambda^{\prime}$ and $\Lambda$ are renormalization parameters and PDF is defined in the infinite momentum frame with $P_{z} \rightarrow \infty$ $[1,2]$. The (quasi-)PDF for negative momentum fraction corresponds to the (quasi-)PDF of the antiparticle. In particular, $Q(-|y|, \Lambda)=-\bar{Q}(|y|, \Lambda)$ with $\bar{Q}$ denoting an antiquark PDF.

Let us first discuss the case that both the quasi-PDF and the PDF are defined in cutoff schemes with UV cutoff $\Lambda^{\prime}$ and $\Lambda$, respectively. The factorization theorem is based on a large momentum expansion in powers of $1 / P_{z}$. The $\mathcal{O}\left(1 / P_{z}^{2}\right)$ correction has a similar convolutional structure to the first term: $\sum_{n=4, \ldots} Z_{n}\left(\Lambda^{\prime}, \Lambda\right) \otimes Q_{n}(\Lambda) / P_{z}^{n-2}$. The scales follow the hierarchy $\Lambda^{\prime} \gg P_{z} \gg \Lambda \gg \Lambda_{\mathrm{QCD}}$, such that the short distance (compared with $1 / \Lambda$ ) physics is encoded in the matching kernels (or Wilson coefficients) $Z$ and $Z_{n}$ while the long distance physics is encoded in the matrix elements $Q$ and $Q_{n}$ with $Q$ of twist-2 and $Q_{n}$ of twist- $n$. In this series, the long and short distance physics are strictly separated by the scale $\Lambda$.

However, the PDFs extracted from experimental data are typically defined in the modified minimal subtraction ( $\overline{\mathrm{MS}}$ ) scheme. This is because the PDFs convolute with Wilson coefficients to form observables in high energy experiments, and it is technically much easier to compute the Wilson coefficients to higher loops in $\overline{\mathrm{MS}}$ than a hard cutoff scheme. However, there is no strict separation between long and short distance physics in the $\overline{\mathrm{MS}}$ scheme. This means the Wilson coefficients could still contain nonperturbative physics called renormalons [132-134] to cause slow convergence in their perturbative expansions.

Mathematically, the renormalon effect could arise when the Borel transform is applied to improve the convergence of a perturbation series. Then an inverse Borel transform is applied after the Borel series is summed. However, sometimes the bad convergence of the series cannot be tamed by the Borel transform but appears as ambiguities in the inverse Borel transform. When $Q$ is defined in the $\overline{\mathrm{MS}}$ scheme with renormalization scale $\mu$ and the quasi-PDF $\tilde{Q}$ is defined in the regularization-independent momentum subtraction (RI/MOM) scheme [13] with scale $\mu^{\prime}$, Eq. (3) can be rewritten as
$\tilde{Q}\left(x, P_{z}, \mu^{\prime}\right)=\int_{-1}^{1} \frac{d y}{|y|} Z\left(\frac{x}{y}, y P_{z}, \mu^{\prime}, \mu\right) Q(y, \mu)+\mathcal{O}\left(\frac{1}{P_{z}^{2}}\right)$.

Since scale separation is not complete in $\overline{\mathrm{MS}}$ and RI/MOM, low energy nonperturbative physics could still exist in $Z$, which slows down the convergence of the perturbative computation of $Z$. It can be shown that by resumming a class of bubble-chain diagrams in $Z$ with the help of the Borel transform, there are ambiguities in the inverse Borel transform that behave like nonperturbative functions $Q_{n} / P_{z}^{n-2}$ with $n \geq 4$.

Since these ambiguities are associated with different choices of the integration paths in the inverse Borel transform, they are not physical. They should be canceled by the $Q_{n}$ terms. Therefore all the $Q_{n}$ 's need to be defined consistently. An independent determination of a higher twist $Q_{n}$ is not meaningful unless the treatment of renormalon ambiguity in the lower twist $Q_{n}$ is specified. Furthermore, one can estimate the size of $Q_{n}$ by the size of the renormalon ambiguity in $Z$. It was through this analysis that Braun, Vladimirov, and Zhang asserted that the leading IR renormalon ambiguity was an $\mathcal{O}\left(\Lambda_{\mathrm{QCD}}^{2} / x^{2} P_{z}^{2}\right)$ effect [6], which was larger than the previous estimation $\mathcal{O}\left(\Lambda_{\mathrm{QCD}}^{2} / P_{z}^{2}\right)$ based on dimensional analysis by a factor of $1 / x^{2}$. Therefore, this result has a big impact to the LaMET program. This motivates us to reinvestigate the renormalon problem in quasi-PDF.

\section{RENORMALON AMBIGUITY IN QUASI-PDFS}

In this section we first briefly review the Borel transform and the possible ambiguity in its inverse transformation. After the discoveries of renormalons [132-134] and further conceptual developments [134-139], higher order behaviors of perturbation series in the context of renormalon ambiguities became relevant phenomenologically [140-142]. (See, e.g., Ref. [143] for a review.) We will compute the renormalon ambiguity caused by the bubblechain diagrams to the quasi-PDF. We will compute the Feynman diagrams in momentum space and then compare our result with the coordinate space computation obtained in Ref. [6].

We start with writing the matching kernel of Eq. (4) as a series expansion in the strong coupling constant $\alpha_{s}$,

$$
Z(\xi)=\delta(1-\xi)+\sum_{n=1}^{\infty}\left(\frac{\alpha_{s}}{4 \pi}\right)^{n} z_{n}(\xi)
$$

where we have only kept the $\xi=x / y$ dependence and dropped the other quantities for brevity. In the $\overline{\mathrm{MS}}$ scheme with renormalization scale $\mu$, 


$$
\alpha_{s}(\mu)=\frac{4 \pi}{\beta_{0} \ln \frac{\mu^{2}}{\Lambda_{\mathrm{QCD}}^{2}}},
$$

where $\beta_{0} \equiv \frac{11}{3} N_{c}-\frac{2}{3} n_{f}, N_{c}$ being the number of colors and $n_{f}$ being the number of quark flavors, and $\Lambda_{\mathrm{QCD}}$ is the strong interaction scale. The series is more convergent after the Borel transform

$$
B[Z(\xi)]=\beta_{0} \delta(1-\xi) \delta(w)+\sum_{n=0}^{\infty}\left(\frac{w}{\beta_{0}}\right)^{n} \frac{z_{n+1}(\xi)}{n !} .
$$

After the Borel series is summed, one can perform an inverse Borel transform back to the original series:

$$
Z(\xi)=\frac{1}{\beta_{0}} \int_{0}^{\infty} d w e^{-\frac{4 \pi w}{\beta_{0} \alpha_{s}}} B[Z(\xi)] .
$$

If the integrant has poles on the real positive $w$ axis, then the above integral will depend on the path of integration on the complex $w$ plane. This uncertainty is called renormalon ambiguity. In an operator product expansion with the $\overline{\mathrm{MS}}$ scheme, the renormalon ambiguities in the lower order Wilson coefficients will be canceled by the higher order matrix elements.

The existence of the renormalon ambiguity is usually demonstrated in the large $n_{f}$ limit with powers of $\left(\alpha_{s} n_{f}\right)$ counted as $\mathcal{O}(1)$ and summed to all orders. Then renormalon ambiguity can arise in the $\alpha_{s}$ expansion of these diagrams. However, it is also well known that QCD is not asymptotically free in the large $n_{f}$ limit. Therefore, even though the finite $n_{f}$ contribution can be formally included by an $1 / n_{f}$ expansion, this expansion is not convergent in QCD. However, if we take the QCD $\beta$ function as a reference, it is probably reasonable to expect that subleading $1 / n_{f}$ corrections are about the same magnitude as the leading order. If this expectation turns out to be true, then the large $n_{f}$ analysis will still be useful. Therefore, we focus on the leading diagrams in the large $n_{f}$ limit to study the renormalon ambiguity in the matching formula between a quasi-PDF and a PDF defined in Eq. (4).

When $\left(\alpha_{s} n_{f}\right)$ is counted as $\mathcal{O}(1)$, the bubble-chain diagrams that dress the gluon propagator in the second row of Fig. 1 are all $\mathcal{O}(1)$. The gray bubble, which is the gluon vacuum polarization, becomes a fermion loop diagram in the large $n_{f}$ limit. We will add the gluon loop contribution to the gluon vacuum polarization as an $1 / n_{f}$ correction as performed in $[6,143]$. This is formally a subleading effect in the $1 / n_{f}$ expansion, but numerically important in QCD - it changes the sign of the QCD beta function from positive to negative. Therefore, the grey bubble in Fig. 1 is the gluon vacuum polarization at oneloop $[132,133,143,144]$

$$
\Pi\left(k^{2}\right)=-\frac{\alpha_{s}(\mu)}{4 \pi} \beta_{0}\left(\ln \frac{-k^{2}}{\mu^{2}}-\frac{5}{3}\right),
$$

where $k$ is the gluon momentum and the $5 / 3$ factor is associated with the $\overline{\mathrm{MS}}$ scheme. Summing the bubble chains yields a factor modifying the gluon propagator:

$$
\frac{1}{1-\Pi\left(k^{2}\right)}=\frac{4 \pi}{\alpha_{s}} \int_{0}^{\infty} \frac{d w}{\beta_{0}} e^{-\frac{4 \pi w}{\beta_{0} \alpha_{s}}+\frac{5}{3} w}\left(\frac{\mu^{2}}{-k^{2}}\right)^{w},
$$

which has a form of an inverse Borel transform with the Borel series $\frac{4 \pi}{\alpha_{s}}\left(\frac{e^{\frac{5}{3}} \mu^{2}}{-k^{2}}\right)^{w}$. We will rewrite it as $\left(\frac{\Lambda_{\mathrm{QCD}}^{2}}{-k^{2}}\right)^{w}$ times a factor which is moved to Eq. (12). Therefore, to compute the diagrams of Fig. 1, one just has to replace the gluon propagator by a dressed one to obtain the Borel series,

$$
\frac{1}{-k^{2}-i \epsilon} \rightarrow \frac{\left(\Lambda_{\mathrm{QCD}}^{2}\right)^{w}}{\left(-k^{2}-i \epsilon\right)^{1+w}}=\frac{\left(\mu^{2} e^{-\frac{4 \pi}{\beta_{0} \alpha_{s}}}\right)^{w}}{\left(-k^{2}-i \epsilon\right)^{1+w}},
$$

where Eq. (6) is used.

Below we list the unpolarized isovector PDF and quasiPDF results of these diagrams. The Borel series of the bubble-chain diagrams in Fig. 1 yields the quark-level quasi-PDF $\tilde{q}(x, \rho)$ [defined similarly to Eq. (2) but with the external hadronic state replaced by a single quark state of momentum $p_{\mu}$,

$$
\begin{aligned}
B[\tilde{q}(x, \rho)]= & 8 \zeta C_{F}\left(\frac{\Lambda_{\mathrm{QCD}}^{2}}{p_{z}^{2}}\right)^{w} e^{\frac{4 \pi w}{\beta_{0} \alpha_{5}}+\frac{5}{3} w} \\
& \times \frac{1}{\sqrt{\pi}} \frac{\Gamma(1 / 2+w)}{\Gamma(1+w)}[\tilde{f}(x, \rho, w)]_{+},
\end{aligned}
$$
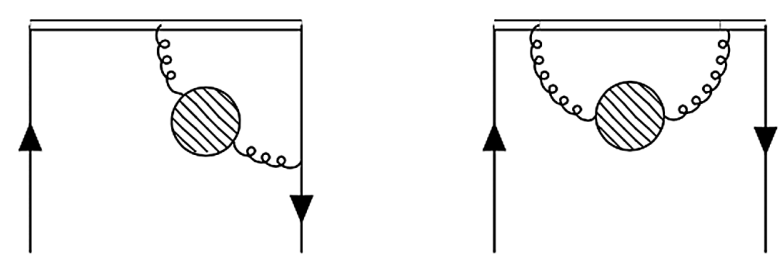

FIG. 1. Bubble-chain diagrams for PDF and quasi-PDF for single quark states. The single lines are quark propagators, curly lines are gluon propagators, and double lines are Wilson lines. The gray bubbles are gluon vacuum polarization diagrams at one-loop. 
where $\rho=-p^{2} / p_{z}^{2}$ is the off-shellness parameter for external quarks. $\zeta$ is the quark spinor factor $\zeta=\sum_{s} \frac{\bar{u}_{s} \gamma^{2} u_{s}}{4 p_{z}}$. $C_{F}=\left(N_{c}^{2}-1\right) / 2 N_{c}$ with $N_{c}$ the number of colors. The plus function

$$
[\tilde{f}(x, \rho, w)]_{+} \equiv \tilde{f}(x, \rho, w)-\delta(x-1) \int_{-\infty}^{\infty} d x^{\prime} \tilde{f}\left(x^{\prime}, \rho, w\right) .
$$

We have computed $\tilde{f}$ up to an integral of the Feynman parameter $y$,

$$
\begin{aligned}
\tilde{f}(x, \rho, w)= & \int_{0}^{1} d y\left\{\left(\frac{1+x^{2}}{1-x}-\frac{\rho}{2(1-x)}\right) \frac{y^{w}}{\left[(x-y)^{2}+y(1-y) \rho\right]^{w+1 / 2}}+\frac{w x(1-y) y^{w-1}}{\left[(x-y)^{2}+y(1-y) \rho\right]^{w+1 / 2}}\right. \\
& -\left(\frac{1}{2 w-1}\right) \frac{1-x}{|1-x|^{1+2 w}}+\frac{1}{2 w-1} \frac{1}{1-x} \frac{w y^{w-1}}{\left[(x-y)^{2}+y(1-y) \rho\right]^{w-1 / 2}} \\
& +\frac{\frac{1}{2}+w}{2(1+w)} \frac{\rho(1-x) y^{w+1}}{\left[(x-y)^{2}+y(1-y) \rho\right]^{w+3 / 2}}-\frac{\left(\frac{3}{2}+w\right)\left(\frac{1}{2}+w\right)}{2(1+w)} \frac{x \rho^{2}(1-y) y^{w+1}}{\left[(x-y)^{2}+y(1-y) \rho\right]^{w+5 / 2}} \\
& \left.-\frac{\frac{1}{2}+w}{2} \frac{\rho x(1-y) y^{w}}{\left[(x-y)^{2}+y(1-y) \rho\right]^{w+3 / 2}}+\left(\frac{1}{2 w-1}\right) \frac{1}{|1-x|^{1+2 w}}\right\} .
\end{aligned}
$$

It is easy to see that for positive and finite $\rho$, if $x$ is different from 0 or 1 and $w$ is different from $1 / 2$ or -1 , then the integrant in the above integral will not diverge. For $x=0,1$, the $y$ integration diverges when $w$ is bigger than a certain value. To see the poles in $w$, one performs the $y$ integration by assuming $w$ is small enough such that the $y$ integration is finite. Then an analytic continuation of $w$ is performed to look for poles in $w$. After this exercise, no additional pole at $x=0,1$ is found. The $w=1 / 2$ pole is corresponding to the UV divergence of the Wilson line selfenergy diagram, which can be subtracted away by the renormalization procedure, such as the RI/MOM scheme used in this work. Therefore we do not consider it further here. The $w=-1$ pole is not on the path of integration of the inverse Borel transform and hence does not cause any ambiguity.

Setting the external quark on-shell $(\rho \rightarrow 0)$, Eq. (14) for the unphysical region $(x>1$ and $x<0)$ becomes

$$
\begin{aligned}
\tilde{f}(x, 0, w)= & \left(\frac{1-x+x^{2}}{1-x}-w x\right) \frac{1}{|x|^{1+2 w}} \frac{1}{1+w}{ }_{2} F_{1}\left(1+w, 1+2 w ; 2+w ; \frac{1}{x}\right)+\frac{x}{|x|^{1+2 w}}{ }_{2} F_{1}\left(w, 1+2 w ; 1+w ; \frac{1}{x}\right) \\
& +\frac{1}{1-x} \frac{1}{|x|^{1+2 w}} \frac{1}{2+w^{2}}{ }_{2} F_{1}\left(2+w, 1+2 w ; 3+w ; \frac{1}{x}\right)+\frac{1}{2 w-1} \frac{1}{|1-x|^{2 w+1}},
\end{aligned}
$$

where ${ }_{2} F_{1}$ is a hypergeometric function. There is no IR renormalon in this region, except the contribution near $x=1$ which yields the delta function in Eq. (20). The UV renormalon at $w=1 / 2$ can be subtracted away by the renormalization procedure discussed above and will not be discussed further.

In the physical region $0<x<1$, IR renormalons exist. They correspond to single poles of $\tilde{f}$ at all positive integer values of $w$ :

$$
\begin{aligned}
\tilde{f}(x, 0, w)= & \left(\frac{1-x+x^{2}}{1-x}-w x\right)\left[-\frac{1+(-1)^{2 w}}{2 x^{w}} \frac{\Gamma(w) \Gamma(1-2 w)}{\Gamma(1-w)}+\left(\frac{-1}{x}\right)^{1+2 w} \frac{1}{1+w^{2}} F_{1}\left(1+w, 1+2 w ; 2+w ; \frac{1}{x}\right)\right] \\
& +\frac{1+(-1)^{2 w}}{2 x^{w}} \frac{\Gamma(1+w) \Gamma(1-2 w)}{\Gamma(1-w)}-\left(\frac{-1}{x}\right)^{2 w} F_{1}\left(w, 1+2 w ; 1+w ; \frac{1}{x}\right) \\
& +\frac{1}{1-x}\left[\frac{1+(-1)^{2 w}}{x^{w-1}} \frac{\Gamma(-2 w) \Gamma(2+w)}{\Gamma(2-w)}+\left(\frac{-1}{x}\right)^{1+2 w} \frac{1}{2+w^{2}} F_{1}\left(2+w, 1+2 w ; 3+w ; \frac{1}{x}\right)\right] \\
& +\frac{1}{2 w-1} \frac{1}{|1-x|^{2 w+1}} .
\end{aligned}
$$

The leading IR renormalon ambiguity in the inverse Borel transform of $\tilde{q}$ is proportional to the residue of the single pole at $w=1$. Equations (15) and (16) yield 


$$
\begin{aligned}
& (1-x)(1-w) \tilde{f}(x, 0, w) \\
& \quad=\theta(x) \theta(1-x)-\frac{(1-w)}{|1-x|^{w}}+\mathcal{O}\left((1-w)^{2}\right)
\end{aligned}
$$

When $w \rightarrow 1$, the term $(1-w) /|1-x|^{w}$ is zero for $x \neq 1$ but undetermined for $x=1$. It is in fact a delta function since

$$
\lim _{w \rightarrow 1} \int_{1^{-}}^{1^{+}} d x \frac{1-w}{|1-x|^{w}}=1
$$

This is consistent with the result of Ref. [145]:

$$
\frac{1-w}{|1-x|^{w}}=\delta(1-x)+\sum_{n=0}^{\infty} \frac{(1-w)^{(n+1)}}{n !}\left(\frac{\ln ^{n}|1-x|}{|1-x|}\right)_{+} .
$$

Equation (17) yields

$$
\lim _{w \rightarrow 1}(1-x)(1-w) \tilde{f}(x, 0, w)=\theta(x) \theta(1-x)-\delta(1-x),
$$

and the leading IR renormalon ambiguity in the inverse Borel transform of $\tilde{q}$ is thus given by

$$
\begin{gathered}
-\left.\frac{\pi}{\beta_{0}} e^{-\frac{4 \pi}{\beta_{0} \alpha_{s}}} \operatorname{Res}[B[\tilde{q}(x), 0]]\right|_{w=1} \\
\quad=\frac{\pi}{\beta_{0}} e^{5 / 3} \frac{\Lambda_{\mathrm{QCD}}^{2}}{p_{z}^{2}} 4 C_{F}\left[\frac{\theta(x) \theta(1-x)-\delta(x-1)}{1-x}\right]_{+}
\end{gathered}
$$

We stress that the IR renormalon is associated with the IR divergence generated when all the particles in the loop become on-shell simultaneously. This is purely an IR effect. Therefore, there is no dependence on the UV regulator in Eq. (21).

After discussing the quasi-PDF, we now turn to the PDF. The quark-level PDF $q\left(x, \frac{\mu^{2}}{-p^{2}}\right)$ is defined similarly to Eq. (1) but with the external hadronic state replaced by a single quark state of momentum $p_{\mu}$. The Borel series of the bubblechain diagrams in Fig. 1 for a quark-level PDF can be obtained by taking $p_{z}$ to infinity before integrating the spatial loop momenta in other directions. The result is

$$
\begin{aligned}
B\left[q\left(x, \frac{\mu^{2}}{-p^{2}}\right)\right]= & 8 \zeta C_{F}\left(\frac{\Lambda_{\mathrm{QCD}}^{2}}{-x(1-x) p^{2}}\right)^{w} e^{\frac{4 \pi w}{\beta_{0} \alpha_{s}}+\frac{5}{3} w} \frac{\Gamma(w+\epsilon)}{\Gamma(1+w)} \\
& \times\left[f\left(x, \epsilon, \frac{\mu^{2}}{-p^{2}}, w\right)\right]_{+}
\end{aligned}
$$

where

$$
\begin{aligned}
f\left(x, \epsilon, \frac{\mu^{2}}{-p^{2}}, w\right)= & \left(\frac{4 \pi \mu^{2}}{-x(1-x) p^{2}}\right)^{\epsilon} x^{w}\{(1-\epsilon)[1-x+w(1-x)-(w+\epsilon)] \\
& \left.+\frac{2 x}{1-x}-\left[\frac{(w+1+\epsilon)(w+\epsilon)}{2(w+1)} \frac{1}{1-x}-\frac{w+\epsilon}{2}-\frac{w+\epsilon}{2(w+1)}\right]\right\}
\end{aligned}
$$

and where the dimensional regularization parameter $\epsilon$ is kept because we want to perform a $w$ expansion later, and this helps to keep the factor $\Gamma(w+\epsilon)$ regulated. The quark-level PDF does not have any IR renormalon ambiguity as there is no pole in the Borel series in the positive $w$ real axis. This result is expected, since the PDF is corresponding to a quasi-PDF in the $p_{z} \rightarrow \infty$ limit and the leading IR renormalon, which is proportional to $1 / p_{z}^{2}$ as shown in Eq. (21), vanishes in this limit.

We can now study how this leading renormalon ambiguity $\delta \tilde{Q}_{\text {ren }}$ affects the quasi-PDF of a hadron, $\tilde{Q}$, through the matching kernel of Eq. (4). The kernel can be converted from Eq. (21) by replacing the quark momentum $p_{z}$ with $y P_{z}, P_{z}$ being the hadron momentum, and replacing $x$ with $x / y$, such that

$$
\begin{aligned}
\delta \tilde{Q}_{\text {ren }}\left(x, P_{z}\right) & =\frac{\pi}{\beta_{0}} e^{5 / 3} C_{F} \frac{\Lambda_{\mathrm{QCD}}^{2}}{P_{z}^{2}} \int_{-1}^{1} \frac{d y}{|y| y^{2}}\left[\frac{\theta\left(1-\frac{x}{y}\right) \theta\left(\frac{x}{y}\right)-\delta\left(1-\frac{x}{y}\right)}{1-\frac{x}{y}}\right]_{+} Q(y) \\
& =\frac{\pi}{\beta_{0}} e^{5 / 3} C_{F} \frac{\Lambda_{\mathrm{QCD}}^{2}}{x^{2} P_{z}^{2}} \int_{|x|}^{1} d \xi\left[\frac{1}{1-\xi}-\delta(1-\xi) \frac{1}{1-\xi}\right]_{+} \xi Q(x / \xi) \\
& =\frac{\pi}{\beta_{0}} e^{5 / 3} C_{F} \frac{\Lambda_{\mathrm{QCD}}^{2}}{x^{2} P_{z}^{2}} \int_{0}^{1} d \xi\left[\frac{1}{1-\xi}-\delta(1-\xi) \frac{1}{1-\xi}\right](\xi Q(x / \xi)-Q(x)) \\
& =\frac{\pi}{\beta_{0}} e^{5 / 3} C_{F} \frac{\Lambda_{\mathrm{QCD}}^{2}}{x^{2} P_{z}^{2}}\left\{\int_{0}^{1} d \xi \frac{1}{1-\xi}[\xi Q(x / \xi)-Q(x)]+Q(x)-x Q^{\prime}(x)\right\},
\end{aligned}
$$




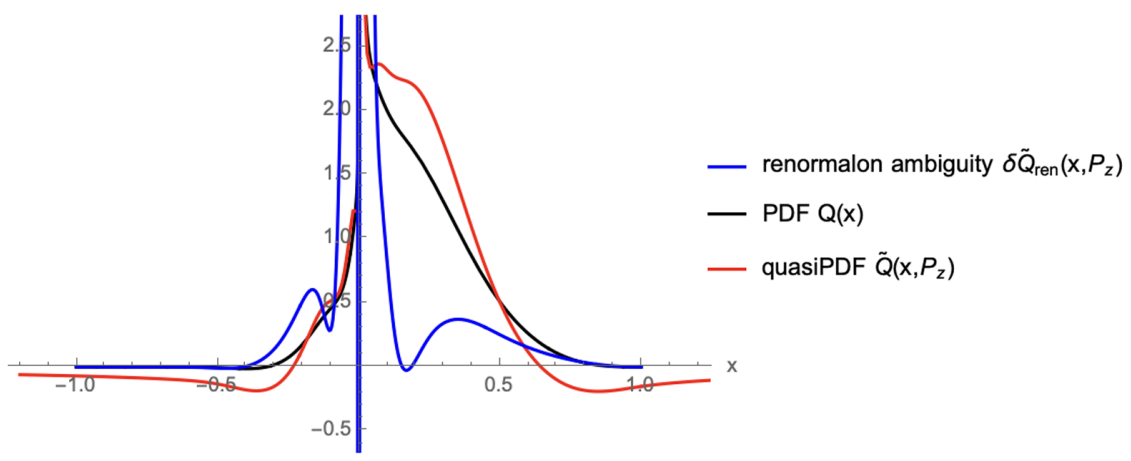

FIG. 2. The leading renormalon ambiguity $\delta \tilde{Q}_{\text {ren }}\left(x, P_{z}\right)$ in the proton isovector quasi-PDF together with the corresponding PDF, $Q(x)$, and the one-loop quasi-PDF $\tilde{Q}\left(x, P_{z}\right)$, all in the $\overline{\mathrm{MS}}$ scheme. $\delta \tilde{Q}_{\mathrm{ren}}\left(x, P_{z}\right)$ is generated with Eq. $(24), P_{z}=1.5 \mathrm{GeV}$, and $Q(x)$ from Ref. [146] (CJ12). $\tilde{Q}$, which is also shown in Fig. 3, is generated by convoluting $Q(x)$ with the one-loop matching kernel. $\delta \tilde{Q}_{\text {ren }}\left(x, P_{z}\right)$ has a large negative contribution near $x=0$ such that the area under the curve is zero. This is a consequence of quark number conservation. The size of $\delta \tilde{Q}_{\text {ren }}\left(x, P_{z}\right)$ is bigger than $\tilde{Q}\left(x, P_{z}\right)$ for almost the entire region of $-0.3 \lesssim x \lesssim 0.1$.

where the integration limit $|x|$ in the second line can be replaced by 0 since $Q(x)$ vanishes outside of $x=[-1,1]$ and we have expanded $(\xi Q(x / \xi)-Q(x)) /(1-\xi)$ around $\xi=1$ before it acts with $\delta(1-\xi)$ in the fourth line.

The plus function in Eq. (24) ensures quark number conservation, such that $\int d x \delta \tilde{Q}_{\text {ren }}=0$. One can show the first two terms in the last line of Eq. (24) cancel after integrating over $x$, and the last two terms are proportional to $\frac{d}{d x}(Q(x) / x)$, which yields vanishing boundary terms after integrating over $x$. Our Eq. (24) is the same as the coordinate space calculation in Ref. [6]. ${ }^{1}$

In Fig. 2, the leading renormalon ambiguity $\delta \tilde{Q}_{\text {ren }}\left(x, P_{z}\right)$ in the proton isovector quasi-PDF is shown, together with the corresponding PDF, $Q(x)$, and the one-loop quasi-PDF $\tilde{Q}\left(x, P_{z}\right)$, all in the $\overline{\mathrm{MS}}$ scheme. Numerically, $\delta \tilde{Q}_{\text {ren }}\left(x, P_{z}\right)$ is a significant effect. Its size is bigger than $\tilde{Q}\left(x, P_{z}\right)$ for almost the entire region of $-0.3 \lesssim x \lesssim 0.1$. In the computation, we have used $Q$ from the proton isovector [i.e., the $(u-d)$ quark combination] PDF extracted by the CTEQJLab Collaboration (CJ12) [146]. $\tilde{Q}$, which is also shown in Fig. 3, is computed with $Q(x)$ convoluted with the one-loop matching kernel. The renormalon ambiguity $\delta \tilde{Q}_{\text {ren }}$ of $\tilde{Q}$ is computed with Eq. (24) with $P_{z}=1.5 \mathrm{GeV}$ and $\Lambda_{\mathrm{QCD}}=0.254 \mathrm{GeV}$. The areas under $Q$ and $\tilde{Q}$ are both one, the isovector charge, while the area under $\delta \tilde{Q}_{\text {ren }}$ is zero, with a large negative contribution near $x=0$. This is the result of fermion number conservation.

The singular behavior near $x=0$ requires the introduction of an IR regulator

$$
Q(x) \rightarrow Q(x) \theta(|x|-|\bar{\epsilon}|)
$$

\footnotetext{
${ }^{1}$ Except our prefactor of $Q^{\prime}(x)$ is $-x$ rather than $-|x|$ as in Ref. [6]. But if one starts from the first line of Eq. (61) of Ref. [6], then the prefactor $Q^{\prime}(x)$ will be $-x$. So this should be a typo of Ref. [6].
}

When $\bar{\epsilon} \rightarrow 0$ is taken at the end of the calculation, the contribution for $|x|<|\bar{\epsilon}|$ becomes $\delta(x)$ with a divergent prefactor. This illusive contribution could easily be overlooked in a numerical analysis. But it is critical for the demonstration of quark number conservation, which is a property of the bubble-chain diagrams that we considered.

Both $\delta \tilde{Q}_{\text {ren }}\left(x, P_{z}\right)$ and $Q(x)$ have support in $[-1,1]$. When $x \rightarrow 0$ or $x \rightarrow 1$,

$$
\delta \tilde{Q}_{\mathrm{ren}}\left(x, P_{z}\right) \propto \frac{\Lambda_{\mathrm{QCD}}^{2}}{x^{2}(1-x) P_{z}^{2}} Q(x),
$$

which is consistent with Ref. [6]. The quasi-PDF $\tilde{Q}\left(x, P_{z}\right)$, however, has support for all values of $x$. Hence $\delta \tilde{Q}_{\text {ren }}$ has no $1 /(1-x)$ enhancement compared to $\tilde{Q}$. Therefore,

$$
\delta \tilde{Q}_{\text {ren }}\left(x, P_{z}\right) \propto \frac{\Lambda_{\mathrm{QCD}}^{2}}{x^{2} P_{z}^{2}} \tilde{Q}\left(x, P_{z}\right) .
$$

Since the renormalon ambiguity discussed above should be canceled by power corrections, one can rewrite Eq. (4) as

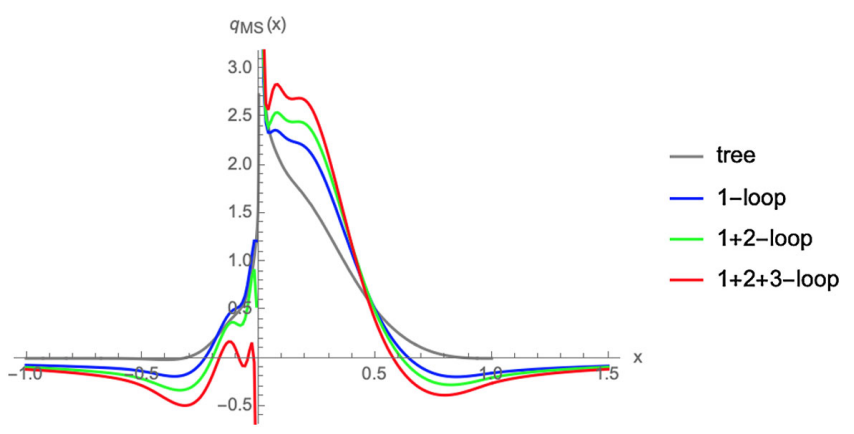

FIG. 3. Isovector proton quasi-PDFs in the $\overline{\mathrm{MS}}$ scheme derived from convoluting the CJ12 proton PDF [146] (shown as the tree level result) with the matching kernels computed with bubblechain diagrams with $\alpha_{s}=0.283, P_{z}=1.5 \mathrm{GeV}$, and $\mu=3 \mathrm{GeV}$. The convergence of the series expansion is slow. 


$$
\begin{aligned}
\tilde{Q}\left(x, P_{z}, \mu^{\prime}\right)= & \int_{-1}^{1} \frac{d y}{|y|} Z\left(\frac{x}{y}, y P_{z}, \mu^{\prime}, \mu\right) Q(y, \mu) \\
& +\mathcal{O}\left(\frac{1}{x^{2} P_{z}^{2}}, \frac{\delta(x)}{P_{z}^{2}}\right)
\end{aligned}
$$

where the first power correction has no $1 /(1-x)$ factor and the second power correction has a divergent prefactor.

\section{REMOVING THE LEADING RENORMALON AMBIGUITY}

Although the bubble-chain diagrams employed in the analysis above might not be numerically the dominant contribution in QCD, we still expect that they will generate all structures of the renormalon ambiguity [140]. Hence Eq. (28) should be quite robust and is expected to be free from the shortcoming of the bubble-chain diagram analysis.

The $R$-scheme proposed in Ref. [147] is designed to remove the leading renormalon ambiguity in a general operator product expansion. In our case, the leading renormalon ambiguity in the matching factor $Z$ and the leading power corrections are both proportional to $1 / P_{z}^{2}$ in Eq. (28). These $1 / P_{z}^{2}$ terms can be removed in the following combination:

$$
\begin{aligned}
\tilde{Q}_{R}\left(x, P_{z}, P_{z}^{\prime}, \Lambda^{\prime}\right) & =\frac{P_{z}^{2} \tilde{Q}\left(x, P_{z}, \Lambda^{\prime}\right)-P_{z}^{\prime 2} \tilde{Q}\left(x, P_{z}^{\prime}, \Lambda^{\prime}\right)}{P_{z}^{2}-P_{z}^{\prime 2}} \\
& =\int_{-1}^{1} \frac{d y}{|y|}\left[\frac{P_{z}^{2} Z\left(\frac{x}{y}, y P_{z}, \Lambda^{\prime}, \mu\right)-P_{z}^{\prime 2} Z\left(\frac{x}{y}, y P_{z}^{\prime}, \Lambda^{\prime}, \mu\right)}{P_{z}^{2}-P_{z}^{\prime 2}}\right] Q(y, \mu)+\mathcal{O}\left(\frac{\alpha_{s} \ln \left(P_{z} / P_{z}^{\prime}\right)}{P_{z}^{2}-P_{z}^{\prime 2}}, \frac{1}{P_{z}^{2} P_{z}^{\prime 2}}\right) .
\end{aligned}
$$

The remaining power corrections can in principle be removed in a similar way as well. The logarithmic term comes from the $\left(\alpha_{s} \ln P_{z}\right) / P_{z}^{2}$ correction in the kernel $Z$ which can be recasted to anomalous dimension $\gamma$ such that the leading renormalon ambiguity in $Z$ scales as $1 / P_{z}^{(2+\gamma)}$. This power can be removed by replacing $2 \rightarrow 2+\gamma$ in the square brackets. Now the remaining power correction is $\mathcal{O}\left(1 / P_{z}^{2} P_{z}^{\prime 2}\right) \sim \mathcal{O}\left(1 / P_{z}^{4}\right)$. So the procedure can be applied again to remove this power correction. However, the computation of the anomalous dimensions beyond the bubble-chain diagrams could be a challenge in removing subleading renormalon ambiguities with this method.

The authors of Ref. [6] also proposed a method to remove the leading renormalon ambiguity by replacing the $\gamma^{z}$ of Eq. (2) with a combination of $\gamma^{z}$ and $\gamma^{t}$. The idea is that the PDF can be extracted using either $\gamma^{z}$ or $\gamma^{t}$ matrix element. However, the leading renormalon ambiguity contributes differently in the two cases. By choosing a specific combination of the $\gamma^{z}$ and $\gamma^{t}$ matrix elements, the leading renormalon ambiguity is largely canceled. However, knowing what combination to use is difficult beyond the bubble-chain approximation. Also, this proposal has another technical issue. On a lattice, the matrix element using $\gamma^{z}$ will mix with another operator of the same dimension that needs to be removed, while the matrix element using $\gamma^{t}$ does not have this problem. Therefore, typically $\gamma^{t}$ is used in lattice computations. However, for the purpose of this work, both of the choices are equivalent since the analysis is performed in the continuum.

\section{BUBBLE-CHAIN CONTRIBUTIONS IN FIXED ORDER PERTURBATIONS}

In the previous section, we have seen that the leading IR renormalon ambiguity of Eq. (24) yields $1 / P_{z}^{2}$ power corrections of Eq. (28) with singular prefactors after summing the bubble-chain diagrams to all orders in $\alpha_{s}$. These $1 / P_{z}^{2}$ terms are canceled in the $R$-scheme by design in Eq. (29). It is natural to ask, if the cancellation works when the series is summed to all orders in $\alpha_{s}$, can the cancellation also happen if the series is truncated to a fixed order? It seems the answer should be yes if the cancellation works for the infinite series and for different values of $\alpha_{s}$ (within the radius of convergence), and then the cancellation ought to happen at each order in $\alpha_{s}$ as well.

To answer this question, we need multiloop corrections to the PDF and quasi-PDF. However, complete results beyond one-loop do not exist yet because of their technical difficulty. Therefore, we resort to large $n_{F}$ expansion again and only include the bubble-chain diagrams. As we will see in Fig. 4(b), the $R$-scheme could indeed introduce a large cancellation of the bubble-chain diagrams at each order in $\alpha_{s}$. This is consistent with what Ref. [147] advocated and demonstrated that the $R$-scheme indeed improved the convergences of QCD perturbation.

Now we show how to extract the fixed order result from the bubble summed result. The bubble-chain contribution to the $(n+1)$ th loop quasi-PDF in the $\overline{\mathrm{MS}}$ scheme can be extracted by expanding the Borel series of Eq. (12) in powers of $w$ : 


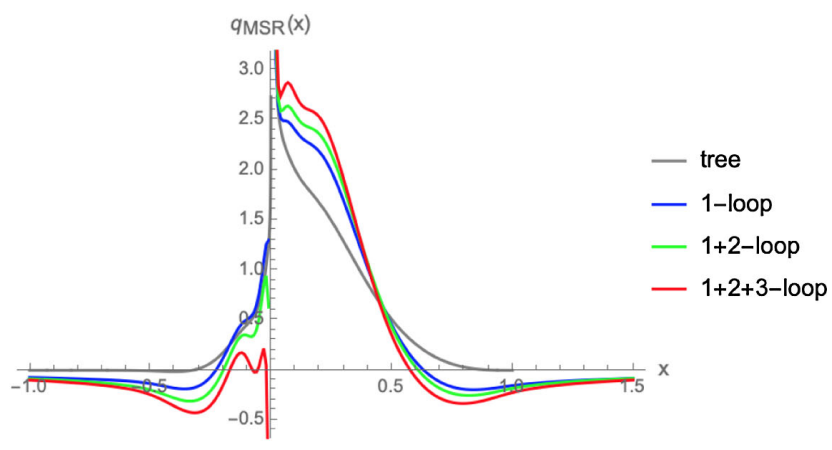

(a)

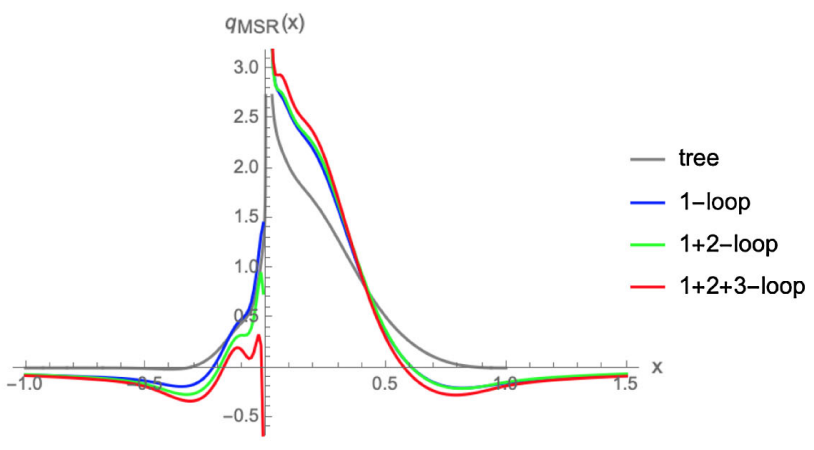

(b)

FIG. 4. Isovector proton quasi-PDFs in $\overline{\mathrm{MS}}$ with the $R$-scheme for (a) $P_{z}^{\prime}=1 \mathrm{GeV}$ and (b) $P_{z}^{\prime}=3 \mathrm{GeV}, \alpha_{s}=0.283, P_{z}=1.5 \mathrm{GeV}$, and $\mu=3 \mathrm{GeV}$. Rapid convergence of the series expansion is seen in (b).

$$
\begin{aligned}
\tilde{q}^{(n+1)}(x, \rho) & =\left.\left(\frac{\alpha_{s}}{4 \pi}\right)^{n+1} \beta_{0}^{n}\left(\frac{d}{d w}\right)^{n} B[\tilde{q}](w)\right|_{w=0} \\
& =8 \zeta C_{F}\left(\frac{\alpha_{s}}{4 \pi}\right)^{n+1} \beta_{0}^{n} \sum_{k=0}^{n}\left(\begin{array}{l}
n \\
k
\end{array}\right)\left(\ln \frac{\mu^{2} e^{5 / 3}}{4 x^{2} p_{z}^{2}}\right)^{n-k} \tilde{C}_{k+1}(x, \rho),
\end{aligned}
$$

where

$$
\tilde{C}_{k+1}(x, \rho)=\left.\frac{d^{k}}{d w^{k}}\left\{\frac{\left(4 x^{2}\right)^{w} \Gamma(1 / 2+w)}{\sqrt{\pi} \Gamma(1+w)}[\tilde{f}(x, \rho, w)]_{+}\right\}\right|_{w=0} .
$$

The bubble-chain contribution to the $(n+1)$ th loop PDF in the $\overline{\mathrm{MS}}$ scheme is

$$
\begin{aligned}
q^{(n+1)}\left(x, \frac{\mu^{2}}{-p^{2}}\right) & =\left.\left(\frac{\alpha_{s}}{4 \pi}\right)^{n+1} \beta_{0}^{n}\left(\frac{d}{d w}\right)^{n} B[q](w)\right|_{w=0} \\
& =8 \zeta C_{F}\left(\frac{\alpha_{s}}{4 \pi}\right)^{n+1} \beta_{0}^{n} \sum_{k=0}^{n}\left(\begin{array}{l}
n \\
k
\end{array}\right)\left(\ln \frac{\mu^{2} e^{5 / 3}}{-(1-x) p^{2}}\right)^{n-k} C_{k+1}\left(x, \frac{\mu^{2}}{-p^{2}}\right),
\end{aligned}
$$

where

$$
C_{k+1}\left(x, \frac{\mu^{2}}{-p^{2}}\right)=\left.\frac{d^{k}}{d w^{k}}\left\{\frac{\Gamma(w+\epsilon)}{x^{w} \Gamma(1+w)}\left[f\left(x, \epsilon, \frac{\mu^{2}}{-p^{2}}, w\right)\right]_{+}\right\}\right|_{w=0, \epsilon \rightarrow 0} .
$$

The results for $\tilde{q}^{(n)}, q^{(n)}$, together with the $\overline{\mathrm{MS}}$ to $\overline{\mathrm{MS}}$ matching kernel

$$
Z^{(n)}\left(\xi, \frac{p_{z}^{2}}{\mu^{2}}\right)=\frac{1}{4 \zeta}\left[\tilde{q}^{(n)}(\xi, \rho \rightarrow 0)-q^{(n)}\left(\xi,-\mu^{2} / p^{2}\right)\right]
$$

for $n=1,2,3$ are listed in Appendix.

These matching kernels are free from IR divergence from diagram-by-diagram cancellations. Note that we should have also included the convolution term $Z^{(1)} \otimes q^{(1)}$ in the $Z^{(2)}$ kernel and the $Z^{(2)} \otimes q^{(1)}+Z^{(1)} \otimes q^{(2)}$ term in the $Z^{(3)}$ kernel were we to include all the $n$-loop diagrams rather than just the bubble-chain diagrams. But here we only investigate how the bubble-chain diagrams which could give rise to the renormalon ambiguities converge at higher loops.

To check the effect of the bubble-chain contribution at the $n$-loop, we convolute the CJ12 proton isovector PDF [146] with $Z^{(n)}$ of Eq. (34) to obtain the corresponding isovector quasi-PDF of the proton at $n$-loop in the $\overline{\mathrm{MS}}$ scheme. The result up to three-loops is shown in Fig. 3. We see that the two-loop contribution is smaller than one-loop but about the same size as the three-loop. Therefore the convergence is already slow at three-loops for the $\overline{\mathrm{MS}}$ quasi-PDF. However, as shown in Fig. 4, the convergence is much better when we use the $R$-scheme of Eq. (29), 
especially when a larger $P_{z}^{\prime}$ is used. The need to use a large $P_{z}^{\prime}$ can be understood because for a fixed order expansion, the kernel only has powers of $\ln P_{z}^{\prime}$ dependence but not $1 / P_{z}^{\prime 2}$ dependence. Hence when we take $P_{z}^{\prime} \rightarrow 0$, the $R$-scheme reduces to the usual $\overline{\mathrm{MS}}$ scheme shown in Fig. 3 and has slow convergence. Therefore, larger $P_{z}^{\prime}$ tends to yield a larger cancellation of the bubble-chain diagrams.

After establishing the power of the $R$-scheme in removing the bubble-chain diagram contributions in two- and three-loop diagrams, we turn to the RI/MOM scheme which is typically used in lattice QCD and see how bubble-chain diagrams contribute in this scheme. In RI/ $\mathrm{MOM}$, all the loop corrections to the matrix element of a single quark state with momentum $p_{\mu}$ are subtracted nonperturbatively at an off-shell kinematics $-p^{2}=\mu_{R}^{2}$ and $p_{z}=p_{R}^{z}$. In momentum space, this amounts to the subtraction [13]

$$
\begin{aligned}
& \tilde{q}_{R}^{(n)}\left(x, \rho, \mu_{R}^{2} /\left(p_{R}^{z}\right)^{2}\right) \\
& \quad=\tilde{q}^{(n)}(x, \rho)-|\eta| \tilde{q}^{(n)}\left(1+\eta(x-1), \mu_{R}^{2} /\left(p_{R}^{z}\right)^{2}\right),
\end{aligned}
$$

with $\eta=p_{z} / p_{R}^{z}$. The RI/MOM renormalized quasi-PDF $\tilde{q}_{R}^{(n)}$ is UV finite, so it does not matter what UV regulator is used to compute $\tilde{q}^{(n)}$ of Eq. (35). The UV regulator will be removed at the end to obtain $\tilde{q}_{R}^{(n)}$. We will just replace $\tilde{q}^{(n)}$ in Eq. (34) by $\tilde{q}_{R}^{(n)}$ to get the $\overline{\mathrm{MS}}$ to RI/MOM matching kernel. It is worth commenting that all the IR renormalon ambiguity comes from the first term in Eq. (35). The second term, the counterterm, takes the external quark off-shell, and hence the Feynman diagrams do not experience IR divergence using the gluon propagator of Eq. (11). Therefore, the subtraction in the RI/MOM scheme does not add additional renormalon ambiguity.

The effect of applying the RI/MOM renormalization to the proton isovector quasi-PDF up to three-loop bubblechain diagrams is shown in Fig. 5. Convergence is seen for

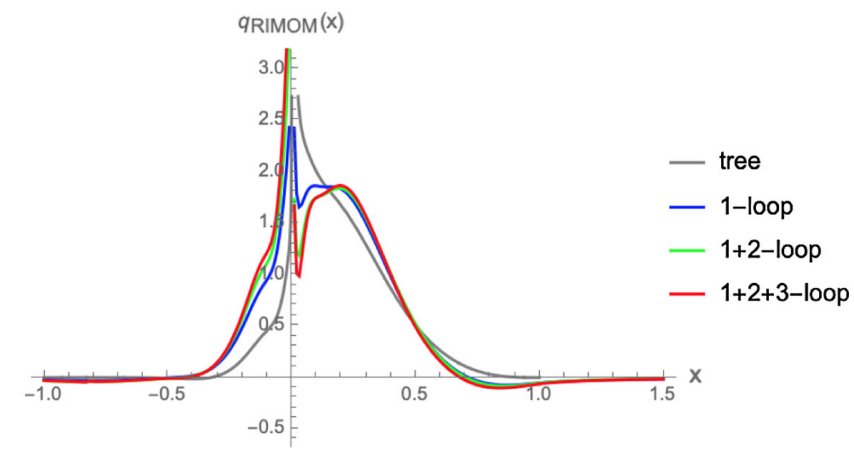

FIG. 5. Isovector proton quasi-PDFs derived from convoluting the proton PDF of Ref. [146] with the matching kernels from bubble-chain diagrams for (a) quasi-PDFs in the $\overline{\mathrm{MS}}$ scheme (b) RI/ MOM renormalized quasi-PDFs, with $\alpha_{s}=0.283, P_{z}=1.5 \mathrm{GeV}$, $\mu=3 \mathrm{GeV}, \mu_{R}=2.4 \mathrm{GeV}$, and $p_{R}^{z}=1.201 \mathrm{GeV}$. the whole range of $x$-the possible slow convergence due to the renormalon effect does not appear up to three-loops in the RI/MOM scheme. Of course, this result is not conclusive for QCD due to the nonconvergence problem of the $1 / n_{f}$ expansion mentioned above. However, the convergence pattern in the large $n_{f}$ world is still interesting on its own. It gives some hope that QCD might have a similar convergence pattern. But one can only know after carrying out the complete higher loop calculations.

Finally, we comment on whether we can take advantage of choosing the BLM scales [148] to improve the convergence of the series expansion. The BLM approach is based on the observation that

$$
\alpha_{\overline{\mathrm{MS}}}\left(\mu e^{-2 D}\right)=\alpha_{\overline{\mathrm{MS}}}(\mu)\left(1+D \beta_{0} \frac{\alpha_{\overline{\mathrm{MS}}}}{\pi}+\cdots\right) .
$$

Hence by choosing a different renormalization scale for each order in the expansion, one can completely remove all the $\alpha_{\overline{\mathrm{MS}}}^{n+1} \beta_{0}^{n}$ dependence in the expansion to speed up the convergence. Interestingly, the $\alpha_{\mathrm{MS}}^{n+1} \beta_{0}^{n}$ dependence is exactly what the bubble-chain diagrams yield [see, e.g., Eq. (30)]. Hence the BLM approach can cancel the renormalon ambiguity from the bubble-chain diagrams to improve the convergence. However, unlike the factor $D$ of Eq. (36) is a constant, the prefactors of our matching kernel depend on the momentum fraction. It is not clear how to absorb them into renormalization scales. Even if we proceed by demanding the bubble-chain diagrams be canceled only for a specific momentum fraction, we still need to benchmark the convergence of this approach with the complete multiloop results which are not yet available. Therefore, we do not pursue this program here in this work.

\section{CONCLUSION}

We have investigated the renormalon ambiguity in the flavor nonsinglet quasi-PDF of a hadron. We follow the usual practice to study the diagrams in the large $n_{f}$ (the number of fermion flavors) limit with powers of $\left(\alpha_{s} n_{f}\right)$ summed to all orders [143]. Although QCD is not asymptotically free in this limit, the qualitative features of the renormalon ambiguity are expected to remain in QCD [140]. Also, taking the $\beta$ function of QCD as a reference, it might be possible that the higher order $1 / n_{f}$ corrections of our calculation are about the same magnitude as the leading order. In that case, our large $n_{f}$ analysis could still be useful although conclusive results can only be drawn with explicit full QCD calculations.

The bubble-chain diagrams were computed in momentum space and the result agreed with the coordinate space computation of Ref. [6] up to one possible typo in a prefactor. We confirmed the assertion of Ref. [6] that the leading IR renormalon ambiguity from bubble-chain diagrams was an $\mathcal{O}\left(\Lambda_{\mathrm{QCD}}^{2} / x^{2} P_{z}^{2}\right)$ effect on the quasi-PDF. 
In addition, we have also found an $\mathcal{O}\left(\delta(x) \Lambda_{\mathrm{QCD}}^{2} / P_{z}^{2}\right)$ term with a divergent prefactor such that quark number conservation is not broken. This ambiguity is supposed to be canceled by power corrections associated with highertwist contributions. Hence, if the power corrections were not included in the analysis, then the error was $\mathcal{O}\left(\Lambda_{\mathrm{QCD}}^{2} / x^{2} P_{z}^{2}, \delta(x) \Lambda_{\mathrm{QCD}}^{2} / P_{z}^{2}\right)$, which is quite significant at small enough $x$, rather than $\mathcal{O}\left(\Lambda_{\mathrm{QCD}}^{2} / P_{z}^{2}\right)$ from dimensional analysis.

To remove this leading IR renormalon ambiguity, we have investigated the proposed $R$-scheme [147], which is designed to cancel all the $\mathcal{O}\left(1 / P_{z}^{2}\right)$ contributions. This cancellation does not rely on the bubble-chain approximation. It is applicable in QCD. Furthermore, since the $R$-scheme cancels the renormalon ambiguity nonperturbatively for different values of $\alpha_{s}$, it is possible that the cancellation also happens at each order in $\alpha_{s}$ to improve the convergence of QCD perturbation [147]. Because the full multiloop results in QCD are not available, we use the bubble-chain diagrams to demonstrate this up to threeloops in Fig. 4(b).

After establishing the power of the $R$-scheme in removing the bubble-chain diagram contributions, we turn to the $\mathrm{RI} / \mathrm{MOM}$ scheme which is typically used in lattice QCD and see how bubble-chain diagrams contribute to the proton isovector quasi-PDF in Fig. 5. Convergence is seen for the whole range of $x$-the possible slow convergence due to the renormalon effect does not appear up to three-loops in the RI/MOM scheme. Of course, this result is not conclusive for QCD due to the nonconvergence problem of the $1 / n_{f}$ expansion mentioned above. But it gives some hope that QCD might have a similar convergence pattern. The final result can only be known after carrying out the complete higher loop calculations.

\section{ACKNOWLEDGMENTS}

We thank Iain Stewart for early involvement in this project and Jian-Hui Zhang for useful discussions. This work is partly supported by the Ministry of Science and Technology, Taiwan, under Grant No. 108-2112-M-002003-MY3 and the Kenda Foundation.

\section{APPENDIX: LOOP EXPANSION OF THE BUBBLE-CHAIN DIAGRAMS}

In this Appendix, we show the $n$th loop correction to the light cone PDF $q^{(n)}$ and quasi-PDF $\tilde{q}^{(n)}$ for a single quark state. We only show the result in the $\overline{\mathrm{MS}}$ scheme. The nonperturbative renormalization of the quasi-PDF in the RI/MOM scheme can be constructed using Eq. (35).

\section{One-loop result}

The one-loop correction to quasi-PDF of a single quark state is

$$
\tilde{q}^{(1)}(x, \rho)=8 \zeta C_{F} \frac{\alpha_{s}}{4 \pi} \tilde{C}_{1}(x, \rho),
$$

where

$$
\begin{aligned}
\tilde{C}_{1}(x, \rho)= & \frac{1}{\sqrt{1-\rho}}\left(\frac{1+x^{2}}{1-x}-\frac{\rho}{2(1-x)}\right) \ln \frac{|1-x|+|x|+\sqrt{1-\rho}}{|1-x|+|x|-\sqrt{1-\rho}}+\frac{x}{|x|}+\frac{|1-x|-|x|}{1-x}-\frac{1}{|1-x|} \\
& -x \frac{(\rho-2+2 x)|x|-(\rho-2 x)|1-x|}{\left(\rho-4 x+4 x^{2}\right)|x(1-x)|}+x \frac{\left(\rho-2 x+2 x^{2}\right)-2|x(1-x)|}{\left(\rho-4 x+4 x^{2}\right)|1-x|} \\
& -\frac{x}{2}\left[\frac{\rho^{2}-4 \rho x+8 x^{2}-8 x^{3}}{\left(\rho-4 x+4 x^{2}\right)^{2}|x|}+\frac{\rho^{2}-4 \rho+4 \rho x+8 x(1-x)^{2}}{\left(\rho-4 x+4 x^{2}\right)^{2}|1-x|}\right] \\
& +\frac{x}{2} \frac{(\rho-2+2 x)|x|+(\rho-2 x)|1-x|}{\left(\rho-4 x+4 x^{2}\right)|x(1-x)|}+\frac{\left(\rho-2 x+2 x^{2}\right)-2|x(1-x)|}{2\left(\rho-4 x+4 x^{2}\right)|1-x|} \\
& -x \frac{\left(\rho-2 x+2 x^{2}\right)-2|x(1-x)|}{\left(\rho-4 x+4 x^{2}\right)|1-x|} \\
= & \begin{cases}\frac{1}{\sqrt{1-\rho}}\left(\frac{1+x^{2}}{1-x}-\frac{\rho}{2(1-x)}\right) \ln \frac{2 x-1+\sqrt{1-\rho}}{2 x-1-\sqrt{1-\rho}}-\frac{\rho}{4 x(x-1)+\rho}+1-\frac{(1-2 x) \rho^{2}}{2(1-x)\left(\rho-4 x+4 x^{2}\right)^{2}} \quad, \quad & x>1 \\
\frac{1}{\sqrt{1-\rho}}\left(\frac{1+x^{2}}{1-x}-\frac{\rho}{2(1-x)}\right) \ln \frac{1+\sqrt{1-\rho}}{1-\sqrt{1-\rho}}-\frac{2 x}{1-x}+\frac{1-2 x}{2(1-x)} & , \quad 0<x<1 . \\
\frac{1}{\sqrt{1-\rho}}\left(\frac{1+x^{2}}{1-x}-\frac{\rho}{2(1-x)}\right) \ln \frac{1-2 x+\sqrt{1-\rho}}{1-2 x-\sqrt{1-\rho}}+\frac{\rho}{4 x(x-1)+\rho}-1+\frac{(1-2 x) \rho^{2}}{2(1-x)\left(\rho-4 x+4 x^{2}\right)^{2}} \quad, & x<0\end{cases}
\end{aligned}
$$


Taking the $\rho \rightarrow 0$ limit and isolating the logarithmic IR divergence, we have

$$
q^{(1)}\left(x,-\mu^{2} / p^{2}\right)=8 \zeta C_{F} \frac{\alpha_{s}}{4 \pi} C_{1}\left(x,-\mu^{2} / p^{2}\right)
$$

where

$$
\begin{aligned}
C_{1}\left(x, \frac{\mu^{2}}{-p^{2}}\right)= & \frac{1+x^{2}}{1-x} \ln \left(\frac{\mu^{2}}{-x(1-x) p^{2}}\right) \\
& -(2-x)+\frac{1-2 x}{2(1-x)} .
\end{aligned}
$$

The one-loop correction to the light cone PDF of a single quark state is nonvanishing only when $0<x<1$, and
The matching kernel for $\tilde{q}^{(1)}(x, \rho \rightarrow 0)$ and $q^{(1)}\left(x,-\mu^{2} / p^{2}\right)$, both in the $\overline{\mathrm{MS}}$ scheme, is

$$
\begin{aligned}
& Z^{(1)}\left(\xi, \frac{p_{z}^{2}}{\mu^{2}}\right)=\frac{1}{4 \zeta}\left[\tilde{q}^{(1)}(\xi, \rho \rightarrow 0)-q^{(1)}\left(\xi,-\mu^{2} / p^{2}\right)\right] \\
& =2 C_{F} \frac{\alpha_{s}}{4 \pi} \begin{cases}\frac{1+\xi^{2}}{1-\xi} \ln \frac{\xi}{\xi-1}+1 & , \quad x>1 \\
\frac{1+\xi^{2}}{1-\xi} \ln \frac{4 \xi(1-\xi) p_{z}^{2}}{\mu^{2}}-\frac{2 \xi}{1-\xi}+2-\xi & , \quad 0<x<1 . \\
\frac{1+\xi^{2}}{1-\xi} \ln \frac{\xi-1}{\xi}-1 & , \quad x<0\end{cases}
\end{aligned}
$$

The IR divergence is canceled between the quasi-PDF and PDF in the matching kernel as we expect that the kernel only compensates the UV difference between the quasi-PDF and PDF.

The kernel has the asymptotic behavior

$$
\left.Z_{1}(\xi)\right|_{\xi \rightarrow \pm \infty}=-\frac{3}{2|\xi|}
$$

with $Z_{n} \equiv Z^{(n)} /\left(2 C_{F}\left(\frac{\alpha_{s}}{4 \pi}\right)^{n} \beta_{0}^{n-1}\right)$. This leading contribution is canceled in the RI/MOM to $\overline{\mathrm{MS}}$ scheme matching which yields better convergence numerically [13].

\section{Two-loop result}

The two-loop correction to quasi-PDF of a single quark state can be written as

$$
\tilde{q}^{(2)}\left(x, \rho, \mu^{2} / p_{z}^{2}\right)=8 \zeta C_{F} \beta_{0}\left(\frac{\alpha_{s}}{4 \pi}\right)^{2}\left[\tilde{C}_{1}(x, \rho) \ln \frac{\mu^{2} e^{5 / 3}}{4 x^{2} p_{z}^{2}}+\tilde{C}_{2}(x, \rho)\right] .
$$

The first term comes from the subdivergence of the gluon vacuum polarization bubble. Taking the limit $\rho \rightarrow 0$ and isolating the logarithmic IR divergence, we have

$$
\tilde{C}_{2}(x, \rho)= \begin{cases}\frac{1+x^{2}}{1-x}\left[\ln \frac{x}{x-1}+\ln ^{2} \frac{x}{x-1}-\mathrm{Li}_{2}\left(\frac{1}{x}\right)\right]+\frac{1}{1-x} & , \quad x>1 \\ \frac{1+x^{2}}{1-x}\left[\frac{1}{2} \ln ^{2} \frac{4}{\rho}-\mathrm{Li}_{2}(x)-\frac{\pi^{2}}{6}-\ln (1-x) \ln \frac{4}{\rho}-\frac{1}{2} \ln ^{2}(1-x)\right] & \\ +\left(\frac{1-2 x}{2(1-x)}-x\right) \ln \frac{4}{\rho}-\frac{3}{2} \frac{1+x}{1-x}+\left(\frac{1}{2(1-x)}+\frac{2 x}{1-x}\right) \ln (1-x) & \\ +\ln x^{2}\left[\frac{1+x^{2}}{1-x} \ln \frac{4}{\rho}-\frac{2 x}{1-x}+\frac{1-2 x}{2(1-x)}\right] & , \\ \frac{1+x^{2}}{1-x}\left[\ln \frac{x-1}{x}-\ln ^{2} \frac{x-1}{x}+\mathrm{Li}_{2}\left(\frac{1}{x}\right)\right]-\frac{1}{1-x} & , \quad x<0\end{cases}
$$


The two-loop light cone PDF is only nonvanishing for $0<x<1$,

$$
q^{(2)}\left(x,-\mu^{2} / p^{2}\right)=8 \zeta C_{F} \beta_{0}\left(\frac{\alpha_{s}}{4 \pi}\right)^{2}\left[C_{1}\left(x,-\mu^{2} / p^{2}\right) \ln \left(\frac{\mu^{2} e^{5 / 3}}{-(1-x) p^{2}}\right)+C_{2}\left(x,-\mu^{2} / p^{2}\right)\right],
$$

where

$$
C_{2}\left(x, \frac{\mu^{2}}{-p^{2}}\right)=\frac{1+x^{2}}{1-x}\left[\frac{\pi^{2}}{12}-\frac{1}{2} \ln ^{2}\left(\frac{\mu^{2}}{-x(1-x) p^{2}}\right)\right]+2(1-x) \ln \left(\frac{\mu^{2}}{-x(1-x) p^{2}}\right)-\frac{3}{2}+x
$$

To this end we will define the matching kernel as

$$
\begin{aligned}
Z^{(2)}\left(\xi, \frac{p_{z}^{2}}{\mu^{2}}\right)= & \frac{1}{4 \zeta}\left[\tilde{q}^{(2)}(\xi, \rho)-\right. \\
& \left.q^{(2)}\left(\xi,-\mu^{2} / p^{2}\right)\right] \\
& =2 C_{F} \beta_{0}\left(\frac{\alpha_{s}}{4 \pi}\right)^{2}\left\{\begin{array}{l}
Z_{1}(\xi) \ln \frac{\mu^{2} e^{5 / 3}}{4 \xi^{2} p_{z}^{2}}+\frac{1+\xi^{2}}{1-\xi}\left[\ln \frac{\xi}{\xi-1}+\ln ^{2} \frac{\xi}{\xi-1}-\operatorname{Li}_{2}\left(\frac{1}{\xi}\right)\right]+\frac{1}{1-\xi}, \quad \xi>1 \\
Z_{1}\left(\xi, \frac{p_{z}^{2}}{\mu^{2}}\right) \ln \frac{\mu^{2} e^{5 / 3}}{4(1-\xi) p_{z}^{2}}+\frac{1+\xi^{2}}{1-\xi}\left[\frac{1}{2} \ln ^{2} \frac{4 \xi(1-\xi) p_{z}^{2}}{\mu^{2}}-\operatorname{Li}_{2}(\xi)-\frac{\pi^{2}}{4}-\frac{1}{2} \ln ^{2}(1-\xi)\right], \\
+2(1-\xi) \ln \frac{4 \xi(1-\xi) p_{z}^{2}}{\mu^{2}}-\frac{3 \xi}{1-\xi}-\xi+\ln (1-\xi), \quad 0<\xi<1 \\
Z_{1}(\xi) \ln \frac{\mu^{2} e^{5 / 3}}{4 \xi^{2} p_{z}^{2}}+\frac{1+\xi^{2}}{1-\xi}\left[\ln \frac{\xi-1}{\xi}-\ln ^{2} \frac{\xi-1}{\xi}+\operatorname{Li}_{2}\left(\frac{1}{\xi}\right)\right]-\frac{1}{1-\xi}, \quad \xi<0
\end{array}\right.
\end{aligned}
$$

which is again free from IR divergence as in the one-loop case. The IR divergence cancellation works diagram by diagram between the quasi-PDF and the PDF. However, we should have also included the convolution term $Z^{(1)} \otimes q^{(1)}$ in the kernel were we to include all the two-loop diagrams rather than just the bubble-chain diagrams.

Again, asymptotically,

$$
\left.Z_{2}(\xi)\right|_{\xi \rightarrow \pm \infty} \sim-\frac{9}{4|\xi|}-\frac{3}{2|\xi|} \ln \frac{\mu^{2} e^{5 / 3}}{4 \xi^{2} p_{z}^{2}}
$$

The kernel will decay faster at large $\xi$ if we renormalize the quasi-PDF with RI/MOM as in the one-loop case.

\section{Three-loop result}

The three-loop quasi-PDF can be written as

$$
\tilde{q}^{(3)}\left(x, \rho, \mu^{2} / p_{z}^{2}\right)=8 \zeta C_{F} \beta_{0}^{2}\left(\frac{\alpha_{s}}{4 \pi}\right)^{3}\left[\tilde{C}_{1}(x, \rho)\left(\ln \frac{\mu^{2} e^{5 / 3}}{4 x^{2} p_{z}^{2}}\right)^{2}+2 \tilde{C}_{2}(x, \rho) \ln \frac{\mu^{2} e^{5 / 3}}{4 x^{2} p_{z}^{2}}+\tilde{C}_{3}(x, \rho)\right],
$$

where the logarithmic dependence in the first two terms come from the subdivergence of the gluon vacuum polarization bubbles. 
Taking the $\rho \rightarrow 0$ limit to isolate the logarithmic IR divergence, we have

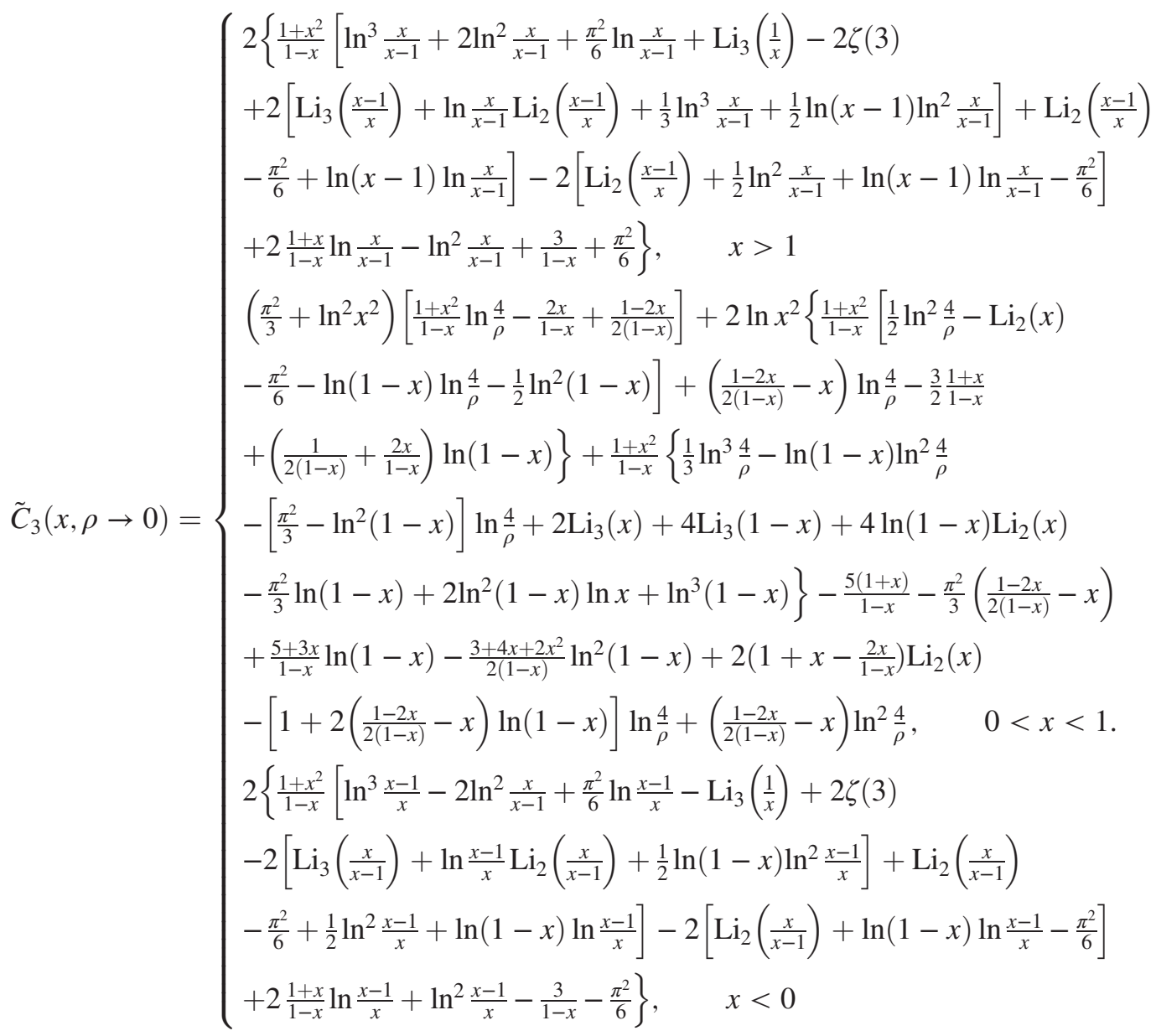

The three-loop PDF is nonvanishing only at $0<x<1$ :

$$
q^{(3)}\left(x, \frac{\mu^{2}}{-p^{2}}\right)=8 \zeta C_{F} \beta_{0}^{2}\left(\frac{\alpha_{s}}{4 \pi}\right)^{3}\left[C_{1} \ln ^{2}\left(\frac{\mu^{2} e^{5 / 3}}{-(1-x) p^{2}}\right)+2 C_{2} \ln \left(\frac{\mu^{2} e^{5 / 3}}{-(1-x) p^{2}}\right)+C_{3}\left(x, \frac{\mu^{2}}{-p^{2}}\right)\right]
$$

where

$$
\begin{aligned}
C_{3}\left(x, \frac{\mu^{2}}{-p^{2}}\right)= & \frac{1+x^{2}}{1-x}\left[\frac{1}{3} \ln ^{3} \frac{\mu^{2}}{-x(1-x) p^{2}}-\frac{\pi^{2}}{6} \ln \frac{\mu^{2}}{-x(1-x) p^{2}}-\frac{2}{3} \zeta(3)\right] \\
& +2(1-x)\left(\ln \frac{\mu^{2}}{-x(1-x) p^{2}}-\ln ^{2} \frac{\mu^{2}}{-x(1-x) p^{2}}\right)+1+\frac{\pi^{2}}{3}(1-x) .
\end{aligned}
$$


Again, we define the kernel without the $Z^{(2)} \otimes q^{(1)}$ and $Z^{(1)} \otimes q^{(2)}$ terms:

$$
\begin{aligned}
& Z^{(3)}\left(\xi, p_{z}, \mu\right)=\frac{1}{4 \zeta}\left[\tilde{q}^{(3)}\left(\xi, p_{z}^{2} / \mu^{2}\right)-q^{(3)}\left(\xi,-\mu^{2} / p^{2}\right)\right]
\end{aligned}
$$

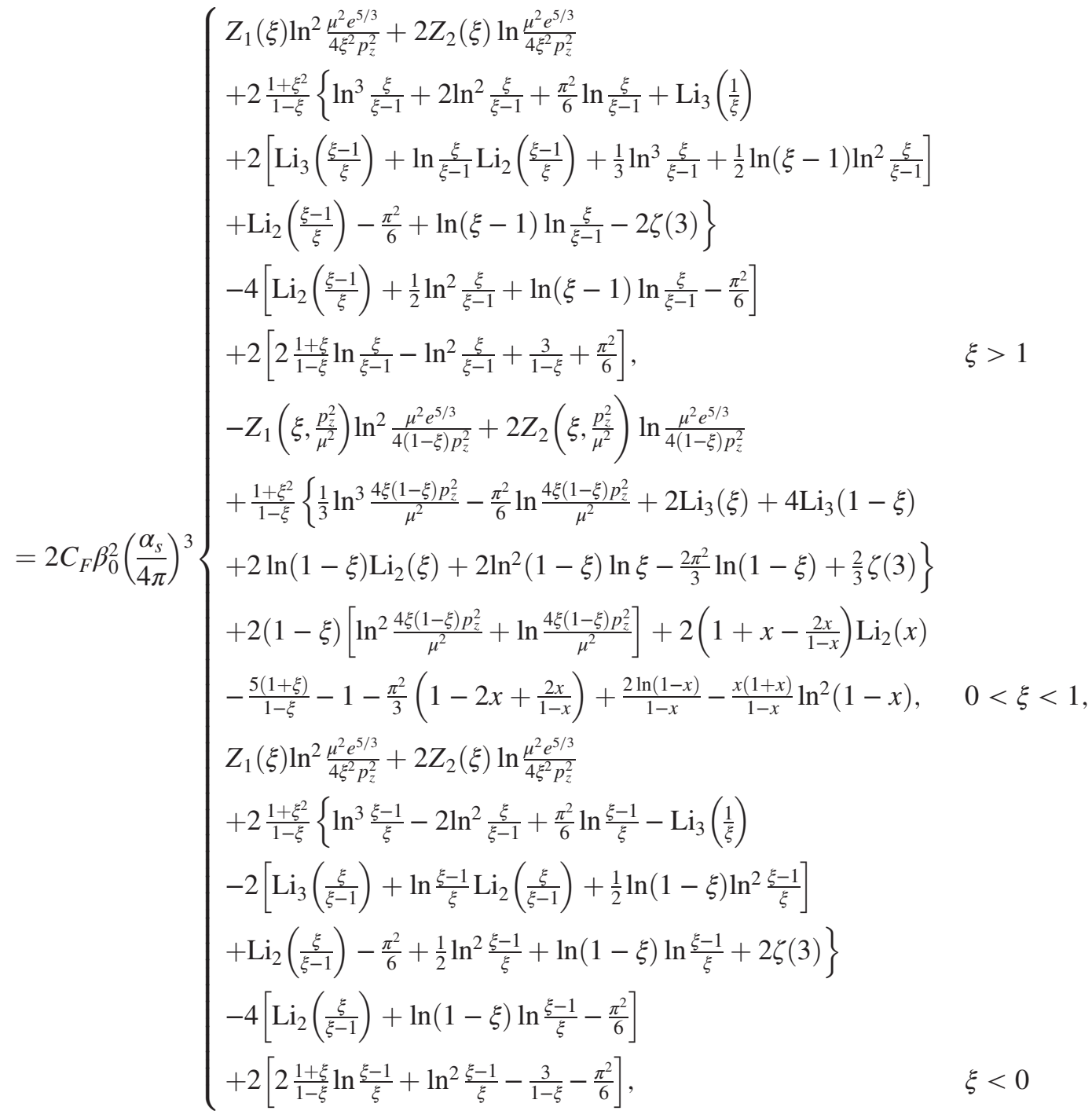

where IR divergence is canceled. And the asymptotic behavior

$$
\left.Z_{3}(\xi)\right|_{\xi \rightarrow \pm \infty} \sim-\frac{27+2 \pi^{2}}{4|\xi|}-\frac{9}{2 \xi} \ln \frac{\mu^{2} e^{5 / 3}}{4 \xi^{2} p_{z}^{2}}-\frac{3}{2|\xi|} \ln ^{2} \frac{\mu^{2} e^{5 / 3}}{4 \xi^{2} p_{z}^{2}}
$$

will again decay faster if RI/MOM renormalization is applied to the quasi-PDF.

[1] X. Ji, Phys. Rev. Lett. 110, 262002 (2013).

[2] X. Ji, Sci. China Phys. Mech. Astron. 57, 1407 (2014).

[3] Y.-Q. Ma and J.-W. Qiu, Phys. Rev. Lett. 120, 022003 (2018).
[4] T. Izubuchi, X. Ji, L. Jin, I. W. Stewart, and Y. Zhao, Phys. Rev. D 98, 056004 (2018).

[5] Y.-S. Liu, W. Wang, J. Xu, Q.-A. Zhang, J.-H. Zhang, S. Zhao, and Y. Zhao, Phys. Rev. D 100, 034006 (2019). 
[6] V. M. Braun, A. Vladimirov, and J.-H. Zhang, Phys. Rev. D 99, 014013 (2019).

[7] X. Xiong, X. Ji, J.-H. Zhang, and Y. Zhao, Phys. Rev. D 90, 014051 (2014).

[8] X. Ji and J.-H. Zhang, Phys. Rev. D 92, 034006 (2015).

[9] X. Ji, A. Schfer, X. Xiong, and J.-H. Zhang, Phys. Rev. D 92, 014039 (2015).

[10] X. Xiong and J.-H. Zhang, Phys. Rev. D 92, 054037 (2015).

[11] X. Ji, J.-H. Zhang, and Y. Zhao, Nucl. Phys. B924, 366 (2017).

[12] C. Monahan, Phys. Rev. D 97, 054507 (2018).

[13] I. W. Stewart and Y. Zhao, Phys. Rev. D 97, 054512 (2018).

[14] M. Constantinou and H. Panagopoulos, Phys. Rev. D 96, 054506 (2017).

[15] J. Green, K. Jansen, and F. Steffens, Phys. Rev. Lett. 121, 022004 (2018).

[16] X. Xiong, T. Luu, and U.-G. Meißner, arXiv:1705.00246.

[17] W. Wang, S. Zhao, and R. Zhu, Eur. Phys. J. C 78, 147 (2018).

[18] W. Wang and S. Zhao, J. High Energy Phys. 05 (2018) 142.

[19] J. Xu, Q.-A. Zhang, and S. Zhao, Phys. Rev. D 97, 114026 (2018).

[20] J.-W. Chen, S. D. Cohen, X. Ji, H.-W. Lin, and J.-H. Zhang, Nucl. Phys. B911, 246 (2016).

[21] J.-H. Zhang, J.-W. Chen, X. Ji, L. Jin, and H.-W. Lin, Phys. Rev. D 95, 094514 (2017).

[22] T. Ishikawa, Y.-Q. Ma, J.-W. Qiu, and S. Yoshida, arXiv:1609.02018.

[23] J.-W. Chen, X. Ji, and J.-H. Zhang, Nucl. Phys. B915, 1 (2017).

[24] X. Ji, J.-H. Zhang, and Y. Zhao, Phys. Rev. Lett. 120, 112001 (2018).

[25] T. Ishikawa, Y.-Q. Ma, J.-W. Qiu, and S. Yoshida, Phys. Rev. D 96, 094019 (2017).

[26] J.-W. Chen, T. Ishikawa, L. Jin, H.-W. Lin, Y.-B. Yang, J.-H. Zhang, and Y. Zhao, Phys. Rev. D 97, 014505 (2018).

[27] C. Alexandrou, K. Cichy, M. Constantinou, K. Hadjiyiannakou, K. Jansen, H. Panagopoulos, and F. Steffens, Nucl. Phys. B923, 394 (2017).

[28] J.-W. Chen, T. Ishikawa, L. Jin, H.-W. Lin, Y.-B. Yang, J.-H. Zhang, and Y. Zhao, arXiv:1710.01089.

[29] H.-W. Lin, J.-W. Chen, T. Ishikawa, and J.-H. Zhang (LP3 Collaboration), Phys. Rev. D 98, 054504 (2018).

[30] J.-W. Chen, T. Ishikawa, L. Jin, H.-W. Lin, A. Schfer, Y.-B. Yang, J.-H. Zhang, and Y. Zhao, arXiv:1711.07858.

[31] H.-N. Li, Phys. Rev. D 94, 074036 (2016).

[32] C. Monahan and K. Orginos, J. High Energy Phys. 03 (2017) 116.

[33] A. Radyushkin, Phys. Lett. B 767, 314 (2017).

[34] G. C. Rossi and M. Testa, Phys. Rev. D 96, 014507 (2017).

[35] C. E. Carlson and M. Freid, Phys. Rev. D 95, 094504 (2017).

[36] R. A. Briceo, J. V. Guerrero, M. T. Hansen, and C. J. Monahan, Phys. Rev. D 98, 014511 (2018).

[37] T. J. Hobbs, Phys. Rev. D 97, 054028 (2018).

[38] Y. Jia, S. Liang, L. Li, and X. Xiong, J. High Energy Phys. 11 (2017) 151.
[39] S.-S. Xu, L. Chang, C. D. Roberts, and H.-S. Zong, Phys. Rev. D 97, 094014 (2018).

[40] Y. Jia, S. Liang, X. Xiong, and R. Yu, Phys. Rev. D 98, 054011 (2018).

[41] G. Spanoudes and H. Panagopoulos, Phys. Rev. D 98, 014509 (2018).

[42] G. Rossi and M. Testa, Phys. Rev. D 98, 054028 (2018).

[43] Y.-S. Liu, J.-W. Chen, L. Jin, H.-W. Lin, Y.-B. Yang, J.-H. Zhang, and Y. Zhao, arXiv:1807.06566.

[44] X. Ji, Y. Liu, and I. Zahed, Phys. Rev. D 99, 054008 (2019).

[45] S. Bhattacharya, C. Cocuzza, and A. Metz, Phys. Lett. B 788, 453 (2019).

[46] A. V. Radyushkin, Phys. Lett. B 788, 380 (2019).

[47] J.-H. Zhang, X. Ji, A. Schfer, W. Wang, and S. Zhao, Phys. Rev. Lett. 122, 142001 (2019).

[48] Z.-Y. Li, Y.-Q. Ma, and J.-W. Qiu, Phys. Rev. Lett. 122, 062002 (2019).

[49] W. Detmold, R. G. Edwards, J. J. Dudek, M. Engelhardt, H.-W. Lin, S. Meinel, K. Orginos, and P. Shanahan (USQCD Collaboration), Eur. Phys. J. A 55, 193 (2019).

[50] R. S. Sufian, C. Egerer, J. Karpie, R. G. Edwards, B. Jo, Y.-Q. Ma, K. Orginos, J.-W. Qiu, and D. G. Richards, arXiv:2001.04960.

[51] C. Shugert, X. Gao, T. Izubichi, L. Jin, C. Kallidonis, N. Karthik, S. Mukherjee, P. Petreczky, S. Syritsyn, and Y. Zhao, Proc. Sci. LATTICE2019 (2019) 269, https://pos .sissa.it/363/269/.

[52] J. R. Green, K. Jansen, and F. Steffens, Phys. Rev. D 101, 074509 (2020).

[53] V. Braun, K. Chetyrkin, and B. Kniehl, arXiv:2004.01043.

[54] H.-W. Lin, Int. J. Mod. Phys. A 35, 2030006 (2020).

[55] M. Bhat, K. Cichy, M. Constantinou, and A. Scapellato, arXiv:2005.02102.

[56] L.-B. Chen, W. Wang, and R. Zhu, Phys. Rev. D 102, 011503 (2020).

[57] X. Ji, arXiv:2003.04478.

[58] L.-B. Chen, W. Wang, and R. Zhu, arXiv:2006.10917.

[59] L.-B. Chen, W. Wang, and R. Zhu, arXiv:2006.14825.

[60] C. Alexandrou, G. Iannelli, K. Jansen, and F. Manigrasso (Extended Twisted Mass Collaboration), arXiv:2007.13800.

[61] Z. Fan, X. Gao, R. Li, H.-W. Lin, N. Karthik, S. Mukherjee, P. Petreczky, S. Syritsyn, Y.-B. Yang, and R. Zhang, arXiv:2005.12015.

[62] X. Ji, Y. Liu, A. Schäfer, W. Wang, Y.-B. Yang, J.-H. Zhang, and Y. Zhao, arXiv:2008.03886.

[63] H.-W. Lin, J.-W. Chen, S. D. Cohen, and X. Ji, Phys. Rev. D 91, 054510 (2015).

[64] C. Alexandrou, K. Cichy, V. Drach, E. Garcia-Ramos, K. Hadjiyiannakou, K. Jansen, F. Steffens, and C. Wiese, Phys. Rev. D 92, 014502 (2015).

[65] C. Alexandrou, K. Cichy, M. Constantinou, K. Hadjiyiannakou, K. Jansen, F. Steffens, and C. Wiese, Phys. Rev. D 96, 014513 (2017).

[66] H.-W. Lin, J.-W. Chen, X. Ji, L. Jin, R. Li, Y.-S. Liu, Y.-B. Yang, J.-H. Zhang, and Y. Zhao, Phys. Rev. Lett. 121, 242003 (2018).

[67] C. Alexandrou, K. Cichy, M. Constantinou, K. Jansen, A. Scapellato, and F. Steffens, Phys. Rev. Lett. 121, 112001 (2018). 
[68] J.-W. Chen, L. Jin, H.-W. Lin, Y.-S. Liu, Y.-B. Yang, J.-H. Zhang, and Y. Zhao, arXiv:1803.04393.

[69] C. Alexandrou, K. Cichy, M. Constantinou, K. Jansen, A. Scapellato, and F. Steffens, Phys. Rev. D 98, 091503 (2018).

[70] H.-W. Lin, J.-W. Chen, X. Ji, L. Jin, R. Li, Y.-S. Liu, Y.-B. Yang, J.-H. Zhang, and Y. Zhao, Phys. Rev. Lett. 121, 242003 (2018).

[71] Z.-Y. Fan, Y.-B. Yang, A. Anthony, H.-W. Lin, and K.-F. Liu, Phys. Rev. Lett. 121, 242001 (2018).

[72] Y.-S. Liu, J.-W. Chen, L. Jin, R. Li, H.-W. Lin, Y.-B. Yang, J.-H. Zhang, and Y. Zhao, arXiv:1810.05043.

[73] W. Wang, J.-H. Zhang, S. Zhao, and R. Zhu, arXiv: 1904.00978.

[74] H.-W. Lin and R. Zhang, Phys. Rev. D 100, 074502 (2019).

[75] K.-F. Liu, arXiv:2007.15075.

[76] R. Zhang, Z. Fan, R. Li, H.-W. Lin, and B. Yoon, Phys. Rev. D 101, 034516 (2020).

[77] J.-W. Chen, L. Jin, H.-W. Lin, Y.-S. Liu, A. Schfer, Y.-B. Yang, J.-H. Zhang, and Y. Zhao, arXiv:1804.01483.

[78] T. Izubuchi, L. Jin, C. Kallidonis, N. Karthik, S. Mukherjee, P. Petreczky, C. Shugert, and S. Syritsyn, Phys. Rev. D 100, 034516 (2019).

[79] X. Gao, L. Jin, C. Kallidonis, N. Karthik, S. Mukherjee, P. Petreczky, C. Shugert, S. Syritsyn, and Y. Zhao, arXiv: 2007.06590.

[80] H.-W. Lin, J.-W. Chen, Z. Fan, J.-H. Zhang, and R. Zhang, arXiv:2003.14128.

[81] Y. Chai et al., arXiv:2002.12044.

[82] S. Bhattacharya, K. Cichy, M. Constantinou, A. Metz, A. Scapellato, and F. Steffens, arXiv:2004.04130.

[83] S. Bhattacharya, K. Cichy, M. Constantinou, A. Metz, A. Scapellato, and F. Steffens, Phys. Rev. D 102, 034005 (2020).

[84] S. Bhattacharya, K. Cichy, M. Constantinou, A. Metz, A. Scapellato, and F. Steffens, arXiv:2006.12347.

[85] Z. Fan, R. Zhang, and H.-W. Lin, arXiv:2007.16113.

[86] R. Zhang, H.-W. Lin, and B. Yoon, arXiv:2005.01124.

[87] J.-H. Zhang, L. Jin, H.-W. Lin, A. Schfer, P. Sun, Y.-B. Yang, R. Zhang, Y. Zhao, and J.-W. Chen (LP3 Collaboration), Nucl. Phys. B939, 429 (2019).

[88] R. Zhang, C. Honkala, H.-W. Lin, and J.-W. Chen, arXiv: 2005.13955.

[89] J.-W. Chen, H.-W. Lin, and J.-H. Zhang, arXiv:1904.12376.

[90] C. Alexandrou, K. Cichy, M. Constantinou, K. Hadjiyiannakou, K. Jansen, A. Scapellato, and F. Steffens, arXiv:2008.10573.

[91] H.-W. Lin, arXiv:2008.12474.

[92] C. Alexandrou, K. Cichy, M. Constantinou, K. Hadjiyiannakou, K. Jansen, A. Scapellato, and F. Steffens, Phys. Rev. D 99, 114504 (2019).

[93] X. Ji, P. Sun, X. Xiong, and F. Yuan, Phys. Rev. D 91, 074009 (2015).

[94] X. Ji, L.-C. Jin, F. Yuan, J.-H. Zhang, and Y. Zhao, arXiv:1801.05930.

[95] M. A. Ebert, I. W. Stewart, and Y. Zhao, Phys. Rev. D 99, 034505 (2019).

[96] M. A. Ebert, I. W. Stewart, and Y. Zhao, J. High Energy Phys. 09 (2019) 037.
[97] M. A. Ebert, I. W. Stewart, and Y. Zhao, J. High Energy Phys. 03 (2020) 099.

[98] X. Ji, Y. Liu, and Y.-S. Liu, Nucl. Phys. B955, 115054 (2020).

[99] X. Ji, Y. Liu, and Y.-S. Liu, arXiv:1911.03840.

[100] M. A. Ebert, S. T. Schindler, I. W. Stewart, and Y. Zhao, arXiv:2004.14831.

[101] P. Shanahan, M. L. Wagman, and Y. Zhao, Phys. Rev. D 101, 074505 (2020).

[102] P. Shanahan, M. Wagman, and Y. Zhao, arXiv:2003.06063.

[103] Q.-A. Zhang et al. (Lattice Parton Collaboration), arXiv: 2005.14572.

[104] K.-F. Liu and S.-J. Dong, Phys. Rev. Lett. 72, 1790 (1994).

[105] W. Detmold and C. Lin, Phys. Rev. D 73, 014501 (2006).

[106] V. Braun and D. Mller, Eur. Phys. J. C 55, 349 (2008).

[107] G. S. Bali et al., Eur. Phys. J. C 78, 217 (2018).

[108] G. S. Bali, V. M. Braun, B. Glle, M. Gckeler, M. Gruber, F. Hutzler, P. Korcyl, A. Schfer, P. Wein, and J.-H. Zhang, Phys. Rev. D 98, 094507 (2018).

[109] W. Detmold, I. Kanamori, C. D. Lin, S. Mondal, and Y. Zhao, Proc. Sci., LATTICE2018 (2018) 106 [arXiv: 1810.12194], https://pos.sissa.it/334/106/pdf.

[110] J. Liang, T. Draper, K.-F. Liu, A. Rothkopf, and Y.-B. Yang (XQCD Collaboration), Phys. Rev. D 101, 114503 (2020).

[111] Y.-Q. Ma and J.-W. Qiu, Phys. Rev. D 98, 074021 (2018).

[112] Y.-Q. Ma and J.-W. Qiu, Int. J. Mod. Phys. Conf. Ser. 37, 1560041 (2015).

[113] A. J. Chambers, R. Horsley, Y. Nakamura, H. Perlt, P. E. L. Rakow, G. Schierholz, A. Schiller, K. Somfleth, R. D. Young, and J. M. Zanotti, Phys. Rev. Lett. 118, 242001 (2017).

[114] A. V. Radyushkin, Phys. Rev. D 96, 034025 (2017).

[115] K. Orginos and A. Radyushkin, J. Karpie, and S. Zafeiropoulos, Phys. Rev. D 96, 094503 (2017).

[116] A. Radyushkin, Phys. Lett. B 781, 433 (2018).

[117] A. Radyushkin, Phys. Rev. D 98, 014019 (2018).

[118] J.-H. Zhang, J.-W. Chen, and C. Monahan, Phys. Rev. D 97, 074508 (2018).

[119] J. Karpie, K. Orginos, and S. Zafeiropoulos, J. High Energy Phys. 11 (2018) 178.

[120] B. Jo, J. Karpie, K. Orginos, A. Radyushkin, D. Richards, and S. Zafeiropoulos, J. High Energy Phys. 12 (2019) 081.

[121] A. V. Radyushkin, Phys. Rev. D 100, 116011 (2019).

[122] B. Jo, J. Karpie, K. Orginos, A. V. Radyushkin, D. G. Richards, R. S. Sufian, and S. Zafeiropoulos, Phys. Rev. D 100, 114512 (2019).

[123] I. Balitsky, W. Morris, and A. Radyushkin, Phys. Lett. B 808, 135621 (2020).

[124] A. Radyushkin, Int. J. Mod. Phys. A 35, 2030002 (2020).

[125] B. Jo, J. Karpie, K. Orginos, A. V. Radyushkin, D. G. Richards, and S. Zafeiropoulos, arXiv:2004.01687.

[126] K. Can et al., arXiv:2007.01523.

[127] H.-W. Lin et al., Prog. Part. Nucl. Phys. 100, 107 (2018).

[128] K. Cichy and M. Constantinou, Adv. High Energy Phys. 2019, 3036904 (2019).

[129] Y. Zhao, Proc. Sci., LATTICE2019 (2020) 267.

[130] X. Ji, Y.-S. Liu, Y. Liu, J.-H. Zhang, and Y. Zhao, arXiv:2004.03543.

[131] X. Ji, arXiv:2007.06613.

[132] D. J. Gross and A. Neveu, Phys. Rev. D 10, 3235 (1974). 
[133] B. Lautrup, Phys. Lett. B 69, 109 (1977).

[134] G. 't Hooft, Subnucl. Ser. 15, 943 (1979).

[135] G. Parisi, Phys. Lett. B 76, 65 (1978).

[136] G. Parisi, Nucl. Phys. B150, 163 (1979).

[137] F. David, Nucl. Phys. B234, 237 (1984).

[138] F. David, Nucl. Phys. B263, 637 (1986).

[139] A. H. Mueller, Nucl. Phys. B250, 327 (1985).

[140] L. S. Brown and L. G. Yaffe, Phys. Rev. D 45, R398 (1992).

[141] V. I. Zakharov, Nucl. Phys. B385, 452 (1992).

[142] A. H. Mueller, in Workshop on QCD: 20 Years Later, Aachen, Germany (1992), https://lib-extopc.kek.jp/preprints/ PDF/1992/9211/9211413.pdf.
[143] M. Beneke, Phys. Rep. 317, 1 (1999).

[144] A. Grozin, in 3rd Dubna International Advanced School of Theoretical Physics (2005), pp. 1-156 [arXiv:hep-ph/ 0508242].

[145] M. D. Schwartz, Quantum Field Theory and the Standard Model (Cambridge University Press, Cambridge, England, 2014).

[146] J. Owens, A. Accardi, and W. Melnitchouk, Phys. Rev. D 87, 094012 (2013).

[147] A. H. Hoang, A. Jain, I. Scimemi, and I. W. Stewart, Phys. Rev. D 82, 011501 (2010).

[148] S. J. Brodsky, G. P. Lepage, and P. B. Mackenzie, Phys. Rev. D 28, 228 (1983). 